\title{
STOCHASTIC LAGRANGIAN METHOD FOR DOWNSCALING PROBLEMS IN COMPUTATIONAL FLUID DYNAMICS
}

\author{
Frédéric Bernardin ${ }^{1}$, Mireille Bossy ${ }^{2}$, Claire Chauvin ${ }^{3}$, Jean-François JabiR $^{4}$ \\ and Antoine Rousseau ${ }^{5}$
}

\begin{abstract}
This work aims at introducing modelling, theoretical and numerical studies related to a new downscaling technique applied to computational fluid dynamics. Our method consists in building a local model, forced by large scale information computed thanks to a classical numerical weather predictor. The local model, compatible with the Navier-Stokes equations, is used for the small scale computation (downscaling) of the considered fluid. It is inspired by Pope's works on turbulence, and consists in a so-called Langevin system of stochastic differential equations. We introduce this model and exhibit its links with classical RANS models. Well-posedness, as well as mean-field interacting particle approximations and boundary condition issues are addressed. We present the numerical discretization of the stochastic downscaling method and investigate the accuracy of the proposed algorithm on simplified situations.
\end{abstract}

Mathematics Subject Classification. 65C20, 65C35, 68U20, 35Q30.

Received July 20, 2009. Revised February 16, 2010.

Published online August 26, 2010.

\section{INTRODUCTION}

In this paper we provide an overview of a new (and currently progressing) approach for the downscaling in Computational Fluid Dynamics (CFD). For numerous practical reasons (computational cost, modelling considerations, etc.), one may be interested in increasing the resolution in a given subdomain, from the simulation of a flow on a larger domain.

Two main issues arise, namely the choice of a local model and its interaction with the largest scales, defining a downscaling model in the considered subdomain. These issues have been widely studied by the community of geophysicists and applied mathematicians as long as deterministic tools are used. Among others, let us quote the Adaptative Mesh Refinement (AMR, [24]) and Large Eddy Simulations (LES, [32]) methods. In all

Keywords and phrases. Langevin models, PDF methods, downscaling methods, fluid dynamics, particle methods.

1 CETE de Lyon, LRPC, Clermont-Ferrand, France. Frederic.Bernardin@developpement-durable.gouv.fr

2 INRIA, TOSCA, Sophia Antipolis, France. Mireille.Bossy@sophia.inria.fr

3 INRIA, MOISE, Grenoble, France. Claire.Chauvin@inria.fr

4 CMM Universidad de Chile, Blanco Encalada 2120, Santiago, Chile. jjabir@dim.uchile.cl

${ }^{5}$ INRIA \& Laboratoire Jean Kuntzmann, 51 rue des Maths, BP 53, 38041 Grenoble Cedex 9, France.

Antoine.Rousseau@inria.fr 


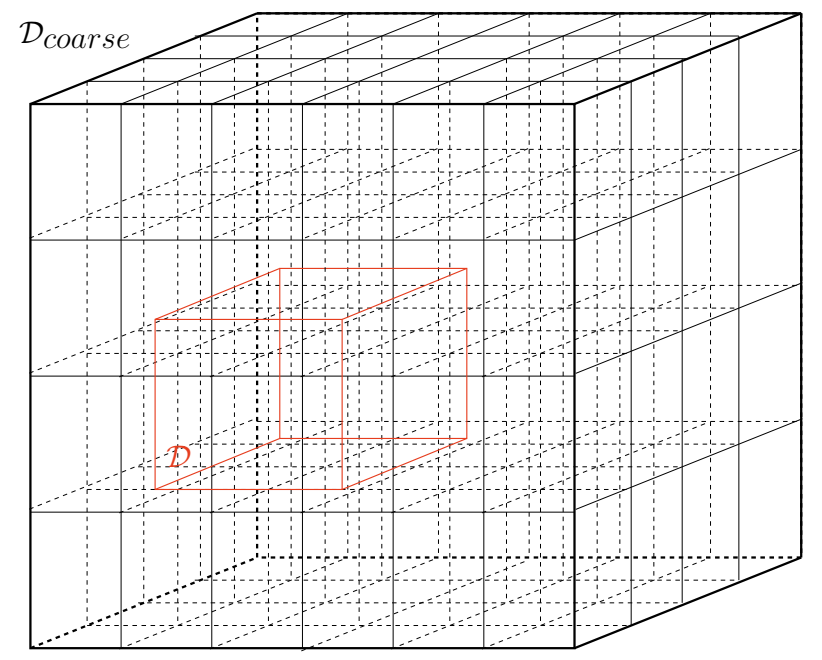

Figure 1. The domain of interest $\mathcal{D}$ is embedded in a wider domain $\mathcal{D}_{\text {coarse }}$.

the deterministic methods, the principal issue relies in the choice of the parameterization that simulates the small scales.

In this work, we consider a new approach for the downscaling in CFD, although the authors are particularly interested in applications to meteorology, in collaboration with physicists (see [1]). The local model that we propose is inspired from Pope's previous works on turbulence (see [25,28]): it consists in modelling the fundamental equations of fluid motion by a stochastic Lagrangian model describing the behaviour of a fluid particle. Because of the both Lagrangian and stochastic nature of our model, it is discretized thanks to an interacting particle system, combining a time Euler scheme for stochastic differential equations and a MonteCarlo approximation method, as we will explain later on. The two main issues that were described above, namely the choice of a local model and the large scale forcing, need to be revisited with the stochastic viewpoint. The objective of this paper is to discuss them.

Let us set the framework: suppose that we have a large-scale model that is run over a wide domain $\mathcal{D}_{\text {coarse }}$ at a given (coarse) resolution (see Fig. 1). Suppose that we are particularly interested on the computation in a subdomain $\mathcal{D} \subset \mathcal{D}_{\text {coarse }}$, but that we want this simulation at a more precise resolution. The objective of a downscaling technique is to use data provided by the large-scale model at the boundary of $\mathcal{D}$, in order to force a local model (to be defined) that will be used for the small-scale simulation in the subdomain $\mathcal{D}$.

Instead of using the well-known Reynolds Averaged Navier Stokes model (1.8), we consider the stochastic Lagrangian model (1.1). We want here to underline the Lagrangian nature of our model. Indeed, contrarily to the deterministic model (1.8), the quantity of interest $\langle U\rangle$ is not computed as the solution of a (system of) $\operatorname{PDE}(\mathrm{s})$, but as the average of particle velocities, the pseudo-fluid particles being subjected to the system of SDEs (1.1). To the best of our knowledge, this approach has never been used as a downscaling technique; it raises several original and difficult problems from the theoretical and numerical viewpoints. The objective of this paper is to discuss them.

The paper is organized as follows. In Section 1, we present the particle model used for the stochastic downscaling method. In particular, we justify the choice of this model thanks to a link with classical $k-\varepsilon$ models. We further discuss the theoretical and numerical issues related to the model: Section 2 is devoted to the theoretical results obtained so far on the model, particularly as far as the boundary conditions are concerned (confinement problem). Well-posedness, as well as mean-field interacting particle approximations and boundary condition issues are addressed. We present in Section 3 the numerical discretization of the downscaling method, and investigate in Section 4 the accuracy of the proposed algorithm on simplified situations. 


\section{Foundations of the stochastic downscaling method (SDM)}

This section is devoted to the description of the Stochastic Downscaling Method, denoted SDM in the sequel, in the framework of turbulence modelling of an incompressible flow with constant mass density. Consider the computational domain $\mathcal{D}$ as an open bounded subset of $\mathbb{R}^{3}$. Along its boundary $\partial \mathcal{D}$, we denote by $U_{\text {ext }}$ a deterministic Eulerian velocity field provided by experimental measures, by statistics or by a numerical weather predictor on a coarser grid. In order to model the flow in $\mathcal{D}$, we consider a couple of stochastic processes $(X, \mathcal{U})$ that respectively describe the location and the velocity of a generic fluid-particle. The evolution of $(X, \mathcal{U})$ is governed by the following system of stochastic differential equations (SDEs):

$$
\left\{\begin{aligned}
X_{t} & =X_{0}+\int_{0}^{t} \mathcal{U}_{s} \mathrm{~d} s \\
\mathcal{U}_{t} & =\mathcal{U}_{0}-\int_{0}^{t} \frac{1}{\varrho} \nabla_{x}\langle\mathcal{P}\rangle\left(s, X_{s}\right) \mathrm{d} s+\int_{0}^{t}\left(\frac{1}{2}+\frac{3}{4} C_{0}\right) \frac{\varepsilon_{L}\left(s, X_{s}\right)}{k_{L}\left(s, X_{s}\right)}\left(\mathbb{E}\left[\mathcal{U}_{s} / X_{s}\right]-\mathcal{U}_{s}\right) \mathrm{d} s \\
& +\int_{0}^{t} \sqrt{C_{0} \varepsilon_{L}\left(s, X_{s}\right)} \mathrm{d} W_{s}+2 \sum_{0<s \leq t}\left(U_{\text {ext }}\left(s, X_{s}\right)-\mathcal{U}_{s^{-}}\right) \mathbb{1}_{\left\{X_{s} \in \partial \mathcal{D}\right\}}, \forall t \in[0, T] .
\end{aligned}\right.
$$

Here $W$ is a standard Brownian motion valued on $\mathbb{R}^{3}, C_{0}$ is a prescribed positive constant, and $\left(X_{0}, \mathcal{U}_{0}\right)$ are random variables whose probability law $\mu_{0}$ is given. The term $\mathbb{E}\left[\mathcal{U}_{t} / X_{t}\right]$ denotes the expected velocity field $\mathcal{U}_{t}$ of particles conditioned by position $X_{t}$. The parameter $\varrho$ is the mass density of the fluid (which is assumed to be constant).

We introduce in the Lagrangian modelling (1.1) the boundary forcing $\sum_{0<s \leq t}\left(U_{\text {ext }}\left(s, X_{s}\right)-\mathcal{U}_{s^{-}}\right) \mathbb{1}_{\left\{X_{s} \in \partial \mathcal{D}\right\}}$ for our downscaling purpose: first it maintains the particle inside $\mathcal{D}$, second it forces the mean of Lagrangian velocities to coincide with the Eulerian data $U_{\text {ext }}(t, x)$ at the boundary. Indeed, when the particle hits the boundary of the computational domain $\mathcal{D}$, its velocity is instantaneously reflected (see Sects. 3 and 4 below) in such a way that the expected velocity satisfies the Dirichlet boundary condition on $\partial \mathcal{D}$ :

$$
\mathbb{E}\left[\mathcal{U}_{t} / X_{t}=x\right]=U_{\text {ext }}(t, x), \quad \text { for } \text { a.e. }(t, x) \in(0, T] \times \partial \mathcal{D} .
$$

We assume that the sum $\sum_{0<s \leq t}\left(U_{\text {ext }}\left(s, X_{s}\right)-\mathcal{U}_{s^{-}}\right) \mathbb{1}_{\left\{X_{s} \in \partial \mathcal{D}\right\}}$ in (1.1) is countable; $k_{L}$ denotes the conditional variance of the particle velocities:

$$
k_{L}(t, x)=\frac{1}{2} \sum_{i=1}^{3}\left(\mathbb{E}\left[\left(\mathcal{U}_{t}^{(i)}\right)^{2} / X_{t}=x\right]-\left(\mathbb{E}\left[\mathcal{U}_{t}^{(i)} / X_{t}=x\right]\right)^{2}\right)
$$

while $\varepsilon_{L}$ is given by

$$
\varepsilon_{L}(t, x)=\frac{C_{\varepsilon}}{\ell_{m}}\left(k_{L}(t, x)\right)^{\frac{3}{2}},
$$

and where $C_{\varepsilon}$ is a positive constant and $\ell_{m}$ is appropriately chosen ${ }^{6}$.

For every $t>0$, we assume that the distribution of $\left(X_{t}, \mathcal{U}_{t}\right)$ admits a probability density with respect to Lebesgue's measure, that is there exists $\rho \in L^{\infty}\left((0, T) ; L^{1}\left(\mathbb{R}^{3} \times \mathbb{R}^{3}\right)\right)$ such that

$$
\mathbb{P}\left(\left(X_{t}, \mathcal{U}_{t}\right) \in(x, x+\mathrm{d} x) \times(u, u+\mathrm{d} u)\right)=\rho(t, x, u) \mathrm{d} x \mathrm{~d} u, \forall t \in(0, T],
$$

where $\mathbb{P}$ stands for the probability measure under which $(X, \mathcal{U}, W)$ are defined. The mass density is a priori defined (up to a multiplicative constant) as the marginal distribution of the position of the fluid-particle:

$$
\varrho(t, x)=C \int_{\mathbb{R}^{3}} \rho(t, x, u) \mathrm{d} u .
$$

\footnotetext{
${ }^{6}$ The so-called mixing length $\ell_{m}$ is a measure of the ability of turbulence to cause mixing. Since the turbulent eddies are limited by the presence of the earth's surface, $\ell_{m}$ may depend on the vertical variable (see [12,34] for more details).
} 
The constant mass density constraint compels the particles to be uniformly distributed in $\mathcal{D}$; namely $\mathbb{P}\left(X_{t} \in\right.$ $(x, x+\mathrm{d} x))$ must be equal to $\mathrm{d} x /|\mathcal{D}|$ where $|\mathcal{D}|$ stands for the Lebesgue measure of $\mathcal{D}$, or equivalently we must have:

$$
\int_{\mathbb{R}^{3}} \rho(t, x, u) \mathrm{d} u=\frac{1}{|\mathcal{D}|}, \forall(t, x) \in(0, T] \times \mathcal{D},
$$

and we recover that $\varrho$ is a constant. As explained in Section 1.2 below, equations (1.1) and (1.5) insure the (mean) free divergence equation

$$
\left(\nabla_{x} \cdot\langle U\rangle\right)=0 \text { on }(0, T] \times \mathcal{D} \text {. }
$$

The Eulerian quantity $\langle\mathcal{P}\rangle$ may be characterized by the following Poisson equation:

$$
-\frac{1}{\varrho} \triangle_{x}\langle\mathcal{P}\rangle(t, x)=\sum_{i, j=1}^{3} \partial_{x_{i}, x_{j}}^{2} \mathbb{E}\left[\mathcal{U}_{t}^{(i)} \mathcal{U}_{t}^{(j)} / X_{t}=x\right] \text { on }(0, T] \times \mathcal{D},
$$

with appropriate boundary condition. Let us notice that, in the case of periodic boundary conditions, equations (1.5) and (1.7) are equivalent (see [3]).

The complete system (1.1), (1.3), subjected to the constraints (1.5), (1.6), defines the SDM model.

Within the domain $\mathcal{D}$ and according to (1.1), the flow undergoes a Langevin based model.

Along the boundary $\partial \mathcal{D}$, the velocity components are submitted to external forcing terms that characterize the downscaling aspect of the model, see (1.2) above.

In the rest of this section, we give a short overview of the link between Eulerian turbulence modelling, (especially the Reynolds Averaged Navier-Stokes (RANS) equations) and the stochastic Lagrangian modelling. We aim to describe the turbulence closure equation in SDM with its equivalent formulation in the RANS model. We highlight the physical meanings of the stochastic Lagrangian SDE's coefficients, in particular $k_{L}$ and $\varepsilon_{L}$ that respectively refer to the turbulent kinetic energy of the system (defined as in (1.10)) and the related (pseudo-)rate of dissipation (see (1.11)), while $\langle\mathcal{P}\rangle$ stands for the mean pressure force acting on the fluid particles.

\subsection{Statistical description of turbulent flows}

We consider an incompressible fluid with constant mass density, with three dimensional velocity $U(t, x)$ and pressure force $\mathcal{P}(t, x)$ acting on the fluid, evaluated at a time $t$ and a location $x$. These quantities are governed by the incompressible Navier-Stokes equations. The statistical description of turbulent flows introduces randomness in the state variables. In this context, the Eulerian fields $U(t, x)$ and $\mathcal{P}(t, x)$ are decomposed into averaged (though macroscopic) fields $\langle U\rangle(t, x)$ and $\langle\mathcal{P}\rangle(t, x)$, and fluctuating parts $u(t, x)$ and $p(t, x)$ (see e.g. [22]). The averaged fields are governed by the so-called Reynolds Averaged Navier-Stokes equations:

$$
\begin{aligned}
\partial_{t}\left\langle U^{(i)}\right\rangle+\left(\langle U\rangle \cdot \nabla_{x}\right)\left\langle U^{(i)}\right\rangle & =-\frac{1}{\varrho} \partial_{x_{i}}\langle\mathcal{P}\rangle+\nu \triangle_{x}\left\langle U^{(i)}\right\rangle-\nabla_{x} \cdot\left\langle u^{(i)} u\right\rangle, \text { on }(0, T) \times \mathbb{R}^{3}, 1 \leq i \leq 3 \\
\left(\nabla_{x} \cdot\langle U\rangle\right) & =0 \text { on }(0, T) \times \mathbb{R}^{3} \\
\langle U\rangle(0, x) & =U_{0}(x)
\end{aligned}
$$

where $\nu$ stands for the viscosity. Note that SDM is more like an Euler system with an artificial viscous dissipation, the value of $\nu$ being implicit in the closure relation (1.3).

These equations are obtained from the original Navier-Stokes system (that we do not recall here) by the formal application of the Reynolds operator $\langle\cdot\rangle$, which consists in computing the average (in the macroscopic sense mentioned above) of the Navier-Stokes equations. Equation (1.8a) corresponds to the mean momentum conservation, and (1.8b) features the mean incompressibility of the flow. The field $U_{0}(x)$ defines the initial averaged state. The Reynolds stress tensor $\left\{\left\langle u^{(i)} u^{(j)}\right\rangle\right\}_{1 \leq i, j \leq 3}$ in (1.8a) denotes the covariance matrix of the velocity field components,

$$
\left\langle u^{(i)} u^{(j)}\right\rangle=\left\langle U^{(i)} U^{(j)}\right\rangle-\left\langle U^{(i)}\right\rangle\left\langle U^{(j)}\right\rangle
$$


and models the stresses due to turbulence motions. As the tensor elements are unknown, the resulting system (1.8) is unclosed. In the same manner, one may derive the equations of the Reynolds stress tensor

$$
\begin{aligned}
\partial_{t}\left\langle u^{(i)} u^{(j)}\right\rangle+\left(\langle U\rangle \cdot \nabla_{x}\left\langle u^{(i)} u^{(j)}\right\rangle\right)+\sum_{l=1}^{3} \partial_{x_{l}}\left\langle u^{(i)} u^{(j)} u^{(l)}\right\rangle= & -\sum_{l=1}^{3}\left(\left\langle u^{(i)} u^{(l)}\right\rangle \partial_{x_{l}}\left\langle U^{(j)}\right\rangle+\left\langle u^{(j)} u^{(l)}\right\rangle \partial_{x_{l}}\left\langle U^{(i)}\right\rangle\right) \\
-\frac{1}{\varrho}\left(\partial_{x_{j}}\left\langle p u^{(i)}\right\rangle+\partial_{x_{i}}\left\langle p u^{(j)}\right\rangle\right)+ & \frac{1}{\varrho}\left(\left\langle p \partial_{x_{j}} u^{(i)}\right\rangle+\left\langle p \partial_{x_{i}} u^{(j)}\right\rangle\right) \\
& +\nu \sum_{l=1}^{3} \partial_{x_{l}}^{2}\left\langle u^{(i)} u^{(j)}\right\rangle-2 \nu \sum_{l=1}^{3}\left\langle\partial_{x_{l}} u^{(i)} \partial_{x_{l}} u^{(j)}\right\rangle,
\end{aligned}
$$

and so on. The complete description of mean flows (especially the velocity moments) requires to solve an infinite cascade of equations. Through years, this closure problem, and the related statistical context, has led to the investigation of turbulence models in order to parameterize the unresolved terms, thanks to laboratory and phenomenological observations, together with physical principles; among them, let us quote Richardson's notion of energy cascade and Kolmogorov's universality laws (see e.g. [28]). For instance, second order closure models parameterize the unknown terms in (1.9) (see [28]). We mention also the general class of the turbulent viscosity models, and the related $k-\varepsilon$-models which close directly the RANS equations (1.8) (see e.g. [22]), introducing further description of turbulence mechanisms brought from the turbulent kinetic energy $k_{E}$ defined by

$$
k_{E}(t, x)=\frac{1}{2} \sum_{i=1}^{3}\left\langle u^{(i)} u^{(i)}\right\rangle(t, x),
$$

and its (pseudo-)rate of dissipation $\varepsilon_{E}$ defined by

$$
\varepsilon_{E}(t, x)=\nu \sum_{i, l=1}^{3}\left\langle\partial_{x_{l}} u^{(i)} \partial_{x_{l}} u^{(i)}\right\rangle(t, x) .
$$

An alternative viewpoint on the RANS equations is proposed by the Lagrangian Probability Density Function (PDF) methods for the modelling and the simulation of turbulent flows. Initiated by Pope [25], the Lagrangian modelling of turbulence flows consists in the description of a general stochastic process $(X, \mathcal{U})$ given at time $t$, the position $X_{t}$ of a fluid particle and its velocity $\mathcal{U}_{t}$.

These particle dynamics are usually referred to as Stochastic Lagrangian Models.

The meaningful point of this Lagrangian approach is that the Eulerian averaged fields are interpreted as the expected velocity value issued from particles conditioned to be located at position $x$. Namely it holds that, for all suitable mapping $g: \mathbb{R}^{3} \rightarrow \mathbb{R}$,

$$
\langle g(U)\rangle(t, x) \simeq \mathbb{E}\left[g\left(\mathcal{U}_{t}\right) / X_{t}=x\right] .
$$

This relation writes, in terms of conditional distribution, as

$$
\langle g(U)\rangle(t, x) \simeq \frac{\int_{\mathbb{R}^{3}} g(u) \rho(t, x, u) \mathrm{d} u}{\int_{\mathbb{R}^{3}} \rho(t, x, u) \mathrm{d} u},
$$

where $\rho$ stands for the probability density (assuming it exists) of the fluid particle at time $t$.

The Lagrangian equations are designed in order to fit with RANS turbulence models. Such a step is achieved by formal developments on the related Fokker-Planck (or forward Kolmogorov) equation. 


\subsection{Turbulence closure equations for SDM}

We now come to the turbulent closure of our model. For $(t, x, u)$ in $(0,+\infty) \times \mathcal{D} \times \mathbb{R}^{3}$, the distribution $\rho(t, x, u)$ of (1.1) may satisfy the Fokker-Planck equation:

$$
\begin{aligned}
\partial_{t} \rho(t, x, u)+\left(u \cdot \nabla_{x} \rho(t, x, u)\right)= & \frac{1}{\varrho}\left(\nabla_{x}\langle\mathcal{P}\rangle(t, x) \cdot \nabla_{u} \rho(t, x, u)\right)-\left(\frac{1}{2}+\frac{3}{4} C_{0}\right) \frac{\varepsilon_{L}(t, x)}{k_{L}(t, x)}\left(\nabla_{u} \cdot(\langle U\rangle(t, x)-u)\right. \\
& \times \rho(t, x, u))+\frac{C_{0} \varepsilon_{L}(t, x)}{2} \triangle_{u} \rho(t, x, u) .
\end{aligned}
$$

For suitable $g: \mathbb{R}^{3} \rightarrow \mathbb{R}$, the function $(t, x) \mapsto \int_{\mathbb{R}^{3}} g(u) \rho(t, x, u) \mathrm{d} u$ satisfies the following equation:

$$
\begin{aligned}
\partial_{t} \int_{\mathbb{R}^{3}} g(u) \rho(t, x, u) \mathrm{d} u+ & \left(\nabla_{x} \cdot \int_{\mathbb{R}^{3}}(u g(u) \rho(t, x, u)) \mathrm{d} u\right)=-\frac{1}{\varrho}\left(\nabla_{x}\langle\mathcal{P}\rangle(t, x) \cdot \int_{\mathbb{R}^{3}} \nabla_{u} g(u) \rho(t, x, u) \mathrm{d} u\right) \\
& +\left(\frac{1}{2}+\frac{3}{4} C_{0}\right) \frac{\varepsilon_{L}(t, x)}{k_{L}(t, x)} \int_{\mathbb{R}^{3}} \nabla_{u} g(u)(\langle U\rangle(t, x)-u) \rho(t, x, u) \mathrm{d} u \\
& +\frac{C_{0} \varepsilon_{L}(t, x)}{2} \int_{\mathbb{R}^{3}} \triangle_{u} g(u) \rho(t, x, u) \mathrm{d} u
\end{aligned}
$$

From this formal equation and the relationship (1.12), we recover the Reynolds turbulence equations. Indeed, taking $g(v):=1$ in $(1.13)$, we get

$$
\partial_{t}\left(\int_{\mathbb{R}^{3}} \rho(t, x, u) \mathrm{d} u\right)+\left(\nabla_{x} \cdot\left(\int_{\mathbb{R}^{3}} \rho(t, x, u) \mathrm{d} u\right)\langle U\rangle(t, x)\right)=0 \text { on }(0, T) \times \mathcal{D},
$$

which, according to the uniform distribution of the particle position (1.5), writes the (mean) free divergence equation (1.6). Next, considering $g(v):=v^{(i)}$ for $1 \leq i \leq 3$, (1.13) yields:

$$
\partial_{t}\left\langle U^{(i)}\right\rangle(t, x)+\left(\nabla_{x} \cdot\left\langle U^{(i)} U\right\rangle(t, x)\right)=-\frac{1}{\varrho} \partial_{x_{i}}\langle\mathcal{P}\rangle(t, x) \text { on }(0, T) \times \mathcal{D},
$$

and thus to the momentum equation (1.8a) when the viscous forces are neglected. Finally, for $1 \leq i, j \leq 3$ and $g(v):=v^{(i)} v^{(j)}$, we obtain the equations for the covariance $\left\langle U^{(i)} U^{(j)}\right\rangle-\left\langle U^{(i)}\right\rangle\left\langle U^{(j)}\right\rangle$. Further using (1.14), we derive the following model for the Reynolds stresses associated to (1.1):

$$
\begin{aligned}
& \partial_{t}\left\langle u^{(i)} u^{(j)}\right\rangle+\left(\langle U\rangle \cdot \nabla_{x}\left\langle u^{(i)} u^{(j)}\right\rangle\right)+\sum_{l=1}^{3} \partial_{x_{l}}\left\langle u^{(i)} u^{(j)} u^{(l)}\right\rangle=-\sum_{l=1}^{3}\left\langle u^{(j)} u^{(l)}\right\rangle \partial_{x_{j}}\left\langle U^{(i)}\right\rangle-\sum_{l=1}^{3}\left\langle u^{(i)} u^{(l)}\right\rangle \partial_{x_{l}}\left\langle U^{(j)}\right\rangle \\
& -2\left(\frac{1}{2}+\frac{3}{4} C_{0}\right) \frac{\varepsilon_{L}}{k_{L}}\left\langle u^{(j)} u^{(i)}\right\rangle+C_{0} \varepsilon_{L} \delta_{i j} \text { on }(0, T) \times \mathcal{D} \text {. }
\end{aligned}
$$

Comparing equation (1.9) with (1.15), one may observe that (1.15) is close to the original Reynolds stress equation. Moreover, this comparison suggests to identify (1.1) as a model for turbulent flows with no direct viscous effects, negligible pressure diffusion $\partial_{x_{j}}\left\langle p u^{(i)}\right\rangle$, and local isotropy. Indeed, in this situation the dissipation tensor $2 \nu \sum_{l=1}^{3}\left\langle\partial_{x_{l}} u^{(i)} \partial_{x_{l}} u^{(j)}\right\rangle$ is reduced to the relation

$$
2 \nu \sum_{l=1}^{3}\left\langle\partial_{x_{l}} u^{(i)} \partial_{x_{l}} u^{(j)}\right\rangle=\frac{2}{3} \varepsilon_{E} \delta_{i j}
$$


where $\delta_{i j}$ is Kronecker's delta and $\varepsilon_{E}(t, x)$ is the pseudo-rate of dissipation given as in (1.11). The remaining terms due to turbulent pressure are closed according to the Rotta's second order closure model (see [28]) which writes

$$
\frac{1}{\varrho}\left(\left\langle p \partial_{x_{j}} u^{(i)}\right\rangle+\left\langle p \partial_{x_{i}} u^{(j)}\right\rangle\right)=-C_{R} \frac{\varepsilon_{E}}{k_{E}}\left\langle u^{(i)} u^{(j)}\right\rangle+\frac{2}{3} C_{R} \varepsilon_{E} \delta_{i j},
$$

for $C_{R}$ denoting the prescribed Rotta's constant. Then, since $k_{E}$ reads as $k_{L},(1.9)$ and (1.15) lead to the identification $\varepsilon_{E}=\varepsilon_{L}$, choosing $C_{0}$ such that $C_{R}=\left(1+\frac{3}{2} C_{0}\right)$. Recalling (1.3), one recovers the HarlowNakayama's $k-\varepsilon$ closure model

$$
\varepsilon_{E}(t, x)=\frac{C_{\varepsilon}}{\ell_{m}}\left(k_{E}(t, x)\right)^{\frac{3}{2}} .
$$

Lagrangian systems are Langevin type equations (see e.g. [13]) extended to the context of turbulent flows. In equation (1.1), the term $-\nabla_{x}\langle\mathcal{P}\rangle\left(t, X_{t}\right) / \varrho$ ensures that the mean incompressibility constraint $(1.8 \mathrm{~b})$ and the constant mass constraint are both satisfied. The coefficient $\left(1 / 2+3 C_{0} / 4\right) \varepsilon_{L} / k_{L}$ models the characteristic time of the drag force $\langle U\rangle\left(t, X_{t}\right)-\mathcal{U}_{t}$. The diffusion part is designed to retrieve - at least heuristically - the steady state predicted by Kolmogorov's universality law (see [28] for further details).

The Lagrangian modelling of turbulent flows has been considered for several closures (see e.g. [26]) and sophisticated turbulent situations (see e.g. [21,28], and references therein). The SDM model is equipped with simple closure models, which nonetheless involve meaningful physical aspects.

Rotta's closure lies in the range of return-to-isotropy model, and is meant to introduce the decay of anisotropy observed in some experiments (see [28]). The Harlow-Nakayama closure model (see e.g. $[15,30]$ ) is a $k-\varepsilon$ closure model commonly used in meteorology. In particular, it introduces a characteristic mixing length scale $\ell_{m}$, which, in the specific case of near-surface turbulence, depends on the vertical coordinate $z$ (see $[12,34]$ ).

\section{ON THE WELL-POSEDNESS OF STOCHASTIC LAGRANGIAN MODELS}

In this section, we review some results obtained by some of the authors on the mathematical study of SDM (see $[2,16]$ ), and more generally on generic problems inherent to Stochastic Lagrangian Models. As illustrated above, equation (1.1) couples several difficulties. On the one hand, the Lagrangian dynamics genuinely stand as SDEs endowing degenerated diffusion terms and singular McKean nonlinearities in the sense that the coefficients depend on the conditional law of the solution. These nonlinearities appear in (1.1) in the form of velocity moments conditioned by the particle position. The Poisson problem (1.7) adds further nonlinearities to the particle dynamics. On the other hand, these difficulties are strengthen by the downscaling application which imposes to introduce appropriate procedure to force boundary conditions of the form (1.2). These original aspects raise the questions of well-posedness, and numerical approximation.

The Lagrangian models considered in this section are in simplified form. Here, the mean pressure term $\langle\mathcal{P}\rangle$, given as solution to (1.7), the mean incompressibility (1.8b) and mass uniformity constraints (1.5), are neglected (these problems being investigated in [3]).

First, we focus on the problem of well-posedness of a Lagrangian system in the free boundary case (i.e. $\mathcal{D}=\mathbb{R}^{d}$ with $d \geq 1$ ). Next, we address the introduction of boundary condition in the specific case of the mean nopermeability boundary (see (2.10) below). Despite these simplifications, the results presented below set the first mathematical bases for the numerical analysis of the particle method proposed for the computation of SDM (1.1) in Section 3.

\subsection{A propagation of chaos result for stochastic Lagrangian models}

In this subsection, we focus on the conditional singularity involved by stochastic Lagrangian models for turbulent flows. We give a well-posedness result for the simplified stochastic Lagrangian model. More precisely, by means of particle approximation, we prove that there exists a process $\left(X_{t}, \mathcal{U}_{t} ; t \in[0, T]\right)$ solution 
to the following SDE

$$
\left\{\begin{array}{l}
X_{t}=X_{0}+\int_{0}^{t} \mathcal{U}_{s} \mathrm{~d} s \\
\mathcal{U}_{t}=\mathcal{U}_{0}+\int_{0}^{t} B\left[X_{s}, \mathcal{U}_{s} ; \rho(s)\right] \mathrm{d} s+\int_{0}^{t} \sigma\left(s, X_{s}, \mathcal{U}_{s}\right) \mathrm{d} W_{s} \\
\rho(t, x, u) \text { is the probability density of }\left(X_{t}, \mathcal{U}_{t}\right) \text { for all } t \in(0, T]
\end{array}\right.
$$

Here, $T$ is a finite horizon time, $\left(X_{0}, \mathcal{U}_{0}\right)$ is distributed according to initial law $\mu_{0}$, and $W$ is a $\mathbb{R}^{d}$-valued Brownian motion. The drift coefficient is the function $B:(x, u, \gamma) \in \mathbb{R}^{d} \times \mathbb{R}^{d} \times L^{1}\left(\mathbb{R}^{2 d}\right) \rightarrow \mathbb{R}^{d}$, defined by

$$
B[x, u ; \gamma]=\left\{\begin{array}{l}
\frac{\int_{\mathbb{R}^{d}} b(v, u) \gamma(x, v) \mathrm{d} v}{\int_{\mathbb{R}^{d}} \gamma(x, v) \mathrm{d} v} \text { if } \int_{\mathbb{R}^{d}} \gamma(x, v) \mathrm{d} v \neq 0, \\
0 \text { elsewhere, }
\end{array}\right.
$$

where $b: \mathbb{R}^{d} \times \mathbb{R}^{d} \rightarrow \mathbb{R}^{d}$ is a bounded continuous interaction kernel. By definition, the drift function $B[x, u ; \rho(s)]$ in (2.1) is (at least formally) a measurable version of the conditional expectation

$$
(x, u) \mapsto \mathbb{E}\left[b\left(\mathcal{U}_{s}, u\right) / X_{s}=x\right]
$$

introduced in Section 1 (see (1.12)). Hypotheses on the diffusion coefficient $\sigma:(0, T) \times \mathbb{R}^{2 d} \rightarrow \mathbb{R}^{d} \times \mathbb{R}^{d}$ are specified below.

Hereafter a solution to (2.1) is understood in the weak sense: there exists a filtered probability space $\left(\Omega, \mathcal{F},\left(\mathcal{F}_{t}\right), \mathbb{P}\right)$ under which a Brownian motion $W$, and a couple of processes $\left(X_{t}, \mathcal{U}_{t} ; t \in[0, T]\right)$ satisfy $(2.1)$. Note that the nonlinearity in the drift term (2.2) imposes to construct a solution which admits time-marginal densities w.r.t. Lebesgue measure, this means that the measure $\mathbb{P} \circ\left(X_{t}, \mathcal{U}_{t}\right)^{-1}$ on $\mathbb{R}^{2 d}$ has a density $\rho(t, x, u)$.

Equivalently, a weak solution to (2.1) can be formulated in terms of a martingale problem: we consider $(\Omega, \mathcal{F})=\left(\mathcal{C}\left([0, T], \mathbb{R}^{2 d}\right), \mathcal{B}\left(\mathcal{C}\left([0, T], \mathbb{R}^{2 d}\right)\right)\right),\left(\left(X_{t}, \mathcal{U}_{t}\right) ; t \in[0, T]\right)$ the canonical processes of $\mathcal{C}\left([0, T], \mathbb{R}^{2 d}\right)$, and $\left(\mathcal{F}_{t}\right)$ the related canonical filtration. A probability measure $\mathbf{P}$ on $\left(\Omega, \mathcal{F},\left(\mathcal{F}_{t}\right)\right)$ is a solution to the martingale problem related to (2.1) if $\mathbf{P}$ satisfies the following:

$(P 1) \mathbf{P} \circ\left(X_{0}, \mathcal{U}_{0}\right)^{-1}=\mu_{0}$, and for all $t \in(0, T], \mathbf{P}_{t}:=\mathbf{P} \circ\left(X_{t}, \mathcal{U}_{t}\right)^{-1}$ admits a positive density $\rho(t, x, u)$ w.r.t. Lebesgue measure.

$(P 2)$ For all $f \in \mathcal{C}_{b}^{2}\left(\mathbb{R}^{2 d}\right)$,

$$
M_{t}:=f\left(X_{t}, \mathcal{U}_{t}\right)-f\left(X_{0}, \mathcal{U}_{0}\right)-\int_{0}^{t} \mathcal{A}_{\rho}(f)\left(s, X_{s}, \mathcal{U}_{s}\right) \mathrm{d} s \text { is a } \mathbf{P} \text {-martingale. }
$$

The operator $\mathcal{A}_{\rho}$ above is the infinitesimal generator of (2.1):

$$
\mathcal{A}_{\rho}(f)(t, x, u):=\left(u \cdot \nabla_{x} f(x, u)\right)+\left(B[x, u ; \rho(t)] \cdot \nabla_{u} f(x, u)\right)+\frac{1}{2} \sum_{i, j=1}^{d} a^{(i, j)}(t, x, u) \partial_{u_{i}, u_{j}}^{2} f(x, u),
$$

where $a:=\sigma \sigma^{*}$ for $\sigma^{*}$ denoting the transpose of $\sigma$. The martingale property $\left(P_{2}\right)$ equivalently writes:

$$
\forall 0 \leq s \leq t \leq T, \mathbb{E}_{\mathbf{P}}\left[\Psi_{s}\left(M_{t}-M_{s}\right)\right]=0,
$$

for all $\Psi_{s}=\prod_{j=1}^{n} \Psi^{j}\left(X_{t_{j}}, \mathcal{U}_{t_{j}}\right)$ given $0 \leq t_{1} \leq \ldots \leq t_{n} \leq s$, and the $\Psi^{j}$ 's are bounded continuous functions on $\mathbb{R}^{2 d}$. From appropriate choices of $f$, one can identify a Brownian motion $W$ such that the canonical process $(X, \mathcal{U})$ solves $(2.1)$ (we refer to $[17,33]$ for a general description on martingale formulation of weak solution). 
Before specifying the hypotheses on the diffusion coefficient $\sigma$ of (2.1), we consider the linear case where $b=0$. In this case, the Lagrangian model writes

$$
\left\{\begin{array}{l}
Y_{t}^{s, y, v}=y+\int_{s}^{t} V_{\theta}^{s, y, v} \mathrm{~d} \theta \\
V_{t}^{s, y, v}=v+\int_{s}^{t} \sigma\left(\theta, Y_{\theta}^{s, y, v}, V_{\theta}^{s, y, v}\right) \mathrm{d} W_{\theta}
\end{array}\right.
$$

and its related backward Kolmogorov equation is

$$
-\partial_{t} \psi(t, x, u)+\frac{1}{2} \sum_{i, j=1}^{d} a^{(i, j)}(t, x, u) \partial_{u_{i}, u_{j}}^{2} \psi(t, x, u)+\left(u \cdot \nabla_{x} \psi(t, x, u)\right)=0 .
$$

Equation (2.5) belongs to the class of ultra-parabolic equations of Kolmogorov type which represents a particular class of parabolic equations with hypoelliptic diffusions. When $a=\eta I_{d}$ for a positive constant $\eta$, the fundamental solution $\Gamma_{\eta}(s, y, v ; t, x, u)$ of $(2.5)$ is the explicit Gaussian probability transition density of the Brownian motion and its primitive $\left(Y^{s, y, v}, V^{s, y, v}\right)$ given by (2.4) with $\sigma=\sqrt{\eta} I_{d}$. Solutions of ultra-parabolic equations and the related properties have been studied in the literature (see [19] for a recent survey). Especially, it has been shown in [10] that (2.5) admits a unique fundamental solution if $a$ satisfies the following hypotheses:

(H1) The matrix $a=\sigma \sigma^{*}$ is bounded and strongly elliptic: there exists $\lambda>0$ such that

$$
\frac{|v|^{2}}{\lambda} \leq \sum_{i, j=1}^{d} a^{(i, j)}(t, x, u) v_{i} v_{j} \leq \lambda|v|^{2}, \forall t \in(0, T],(x, u) \in \mathbb{R}^{2 d}, v \in \mathbb{R}^{d} .
$$

(H2) For all $1 \leq i, j \leq d$, there exists $\alpha \in(0,1]$ and $K>0$ such that, for all $(s, y, v),(t, x, u) \in(0, T] \times \mathbb{R}^{2 d}$,

$$
\left|a^{(i, j)}(t, x, u)-a^{(i, j)}(s, y, v)\right| \leq K\left(|t-s|^{\frac{\alpha}{2}}+|x-y-(t-s) v|^{\frac{\alpha}{3}}+|u-v|^{\alpha}\right) .
$$

Some properties of the fundamental solution shown in [10,11], combined with the Feynman-Kac's formula for the backward Kolmogorov equation associated to (2.5), allow one to prove the following lemma.

Lemma 2.1. Under Assumptions (H1)-(H2), for all $(s, y, v) \in[0, T) \times \mathbb{R}^{2 d}$ there exists a solution $\left(Y^{s, y, v}, V^{s, y, v}\right)$ to (2.4). In addition, this solution admits a density $\Gamma(s, y, v ; t, x, u)$ w.r.t. Lebesgue measure, for all $0 \leq s<t$, such that $\nabla_{v} \Gamma(s, y, v ; t, x, u)$ exists and is continuous. Moreover, there exist $C, c>0$ and $\eta>0$ such that

$$
\begin{aligned}
& \int_{\mathbb{R}^{2 d}}\left|\nabla_{v} \Gamma(s, y, v ; t, x, u)\right| \mathrm{d} x \mathrm{~d} u \leq \frac{C}{\sqrt{t-s}}, \forall 0 \leq s<t \leq T,(y, v) \in \mathbb{R}^{2 d}, \\
& \Gamma(s, y, v ; t, x, u) \geq c \Gamma_{\eta}(s, y, v ; t, x, u), \forall 0 \leq s<t \leq T,(y, v) \in \mathbb{R}^{2 d} .
\end{aligned}
$$

Coming back to the solution $(X, \mathcal{U})$ to $(2.1)$ (if it exists), the Girsanov theorem (removing the drift $B$ in $(2.1)$ by a change of probability argument) allows one to prove the absolute continuity of the probability law of $\left(X_{t}, \mathcal{U}_{t}\right)$ w.r.t. the Lebesgue measure and the a priori positivity of its density. Additionally, we identify the time-marginal densities of the law of $(X, \mathcal{U})$ as the unique solution of a mild equation. This further shows the uniqueness of a solution to (2.1) (see [2] for details).

We construct a solution to the equation (2.1) using a particle approximation procedure, inspired from the classical framework of nonlinear SDEs of McKean type and their approximation by a particle system in weak and mean field interaction. 
Equation (2.1) is a singular case of nonlinear McKean equation. The nonlinearity is of conditional form, and the diffusion coefficient is partially degenerated. Following the lines of the propagation of chaos theory, we introduce the following interacting particle system $\left\{\left(X^{i, \delta, N_{p}}, \mathcal{U}^{i, \delta, N_{p}}\right) ; 1 \leq i \leq N_{p}\right\}$ satisfying:

$$
\left\{\begin{array}{l}
X_{t}^{i, \delta, N_{p}}=X_{0}^{i}+\int_{0}^{t} \mathcal{U}_{s}^{i, \delta, N_{p}} \mathrm{~d} s, \\
\mathcal{U}_{t}^{i, \delta, N_{p}}=\mathcal{U}_{0}^{i}+\int_{0}^{t} \frac{\frac{1}{N_{p}} \sum_{j=1}^{N_{p}} b\left(\mathcal{U}_{s}^{j, \delta, N_{p}}, \mathcal{U}_{s}^{i, \delta, N_{p}}\right) \phi_{\delta}\left(X_{s}^{i, \delta, N_{p}}-X_{s}^{j, \delta, N_{p}}\right)}{\frac{1}{N_{p}} \sum_{j=1}^{N_{p}} \phi_{\delta}\left(X_{s}^{i, \delta, N_{p}}-X_{s}^{j, \delta, N_{p}}\right)+\delta} \mathrm{d} s+\int_{0}^{t} \sigma\left(s, X_{s}^{i, \delta, N_{p}}, \mathcal{U}_{s}^{i, \delta, N_{p}}\right) \mathrm{d} W_{s}^{i},
\end{array}\right.
$$

where $\left\{\left(X_{0}^{i}, \mathcal{U}_{0}^{i}\right), W^{i} ; i \geq 1\right\}$ are independent copies of $\left(\left(X_{0}, \mathcal{U}_{0}\right), W\right)$, and where $\left\{\phi_{\delta}:=1 / \delta^{d} \phi(x / \delta) ; \delta>0\right\}$ is a family of mollifiers.

Roughly speaking, a particle system like (2.6) propagates chaos (or is $P$-chaotic) if, when the number of particles $N_{p}$ goes to infinity, any finite subsystem of particles tends to behave like a system of independent particles, each one having the same law $P$ (we refer to [35] for details).

We define the smoothed kernel $B_{\delta}[x, u ; \gamma]$, for all $(x, u) \in \mathbb{R}^{2 d}$ and for all nonnegative $\gamma \in L^{1}\left(\mathbb{R}^{2 d}\right)$, by

$$
B_{\delta}[x, u ; \gamma]=\frac{\int_{\mathbb{R}^{d}} b(v, u) \phi_{\delta} \star \gamma(x, v) \mathrm{d} v}{\int_{\mathbb{R}^{d}} \phi_{\delta} \star \gamma(x, v) \mathrm{d} v+\delta},
$$

with $\phi_{\delta} \star \gamma$ denoting the convolution product between $\phi_{\delta}$, and $\gamma$ along $x$-variable: $\phi_{\delta} \star \gamma(x, u)=\int_{\mathbb{R}} \phi_{\delta}(x-$ y) $\gamma(y, u) \mathrm{d} y$. Considering the empirical measure $\mu^{\delta, N_{p}}:=1 / N_{p} \sum_{j=1}^{N_{p}} \delta_{\left\{X^{j, \delta, N_{p}}, \mathcal{U}^{\left.j, \delta, N_{p}\right\}}\right.}$ (for $\delta_{\{\cdot\}}$ the Dirac measure) of the particle system, one can equivalently write equation (2.6) as

$$
\left\{\begin{array}{l}
X_{t}^{i, \delta, N_{p}}=X_{0}^{i}+\int_{0}^{t} \mathcal{U}_{s}^{i, \delta, N_{p}} \mathrm{~d} s \\
\mathcal{U}_{t}^{i, \delta, N_{p}}=\mathcal{U}_{0}^{i}+\int_{0}^{t} B_{\delta}\left[X_{s}^{i, \delta, N_{p}}, \mathcal{U}_{s}^{i, \delta, N_{p}} ; \mu_{s}^{\delta, N_{p}}\right] \mathrm{d} s+\int_{0}^{t} \sigma\left(s, X_{s}^{i, \delta, N_{p}}, \mathcal{U}_{s}^{i, \delta, N_{p}}\right) \mathrm{d} W_{s}^{i},
\end{array}\right.
$$

where we have naturally extended the definition of the kernel $B_{\delta}$ to the (time-marginal) measures $\left(\mu_{t}^{\delta, N_{p}}\right.$, $t \in[0, T])$.

Under Assumptions $(H 1)-(H 2)$ and owing to the boundedness of $b$, the well-posedness of a particle system solution to $(2.6)$, defined on some filtered probability space $\left(\Omega, \mathcal{F},\left(\mathcal{F}_{t}\right), \mathbb{P}\right)$, is derived from the case $b=0$ via a change of probability argument and the Girsanov theorem. The asymptotic behavior of the $N_{p}$-particle system (2.6) yields to the following result.

Theorem 2.2. Assume that $(H 1)-(H 2)$ hold true, and that the law $\mu_{0}$ of $\left(X_{0}, \mathcal{U}_{0}\right)$ satisfies

$$
\int_{\mathbb{R}^{2 d}}\left(|x|+|u|^{2}\right) \mu_{0}(\mathrm{~d} x \mathrm{~d} u)<+\infty .
$$

Then, for fixed $\delta>0$, the particle system $(2.6)$ is $\mathbf{P}^{\delta}$-chaotic: for $\phi_{1}, \ldots, \phi_{k} \in \mathcal{C}_{b}\left(\mathcal{C}_{b}\left([0, T] ; \mathbb{R}^{2 d}\right) ; \mathbb{R}\right), k>1$,

$$
\lim _{N_{p} \rightarrow+\infty} \mathbb{E}_{\mathbb{P}}\left[\phi_{1}\left(X^{1, \delta, N_{p}}, \mathcal{U}^{1, \delta, N_{p}}\right) \ldots \phi_{k}\left(X^{k, \delta, N_{p}}, \mathcal{U}^{k, \delta, N_{p}}\right)\right]=\prod_{i=1}^{k} \mathbb{E}_{\mathbf{P}^{\delta}}\left[\phi_{i}(X, \mathcal{U})\right],
$$

where $\left(\left(X_{t}, \mathcal{U}_{t}\right) ; t \in[0, T]\right)$ are the canonical processes of $\mathcal{C}\left([0, T], \mathbb{R}^{2 d}\right)$ and $\mathbf{P}^{\delta}$ is the unique probability measure solution to the following martingale problem: 
$\left(P 1^{\delta}\right) \mathbf{P}^{\delta} \circ\left(X_{0}, \mathcal{U}_{0}\right)^{-1}=\mu_{0}$, and, for all $t \in(0, T], \mathbf{P}_{t}^{\delta}:=\mathbf{P}^{\delta} \circ\left(X_{t}, \mathcal{U}_{t}\right)^{-1}$ admits a (Lebesgue) density $\rho^{\delta}(t, x, u)$.

$\left(P 2^{\delta}\right)$ For all $f \in \mathcal{C}_{b}^{2}\left(\mathbb{R}^{2 d}\right)$,

$$
M_{t}^{\delta}:=f\left(X_{t}, \mathcal{U}_{t}\right)-f\left(X_{0}, \mathcal{U}_{0}\right)-\int_{0}^{t} \mathcal{A}_{\rho^{\delta}}^{\delta}(f)\left(s, X_{s}, \mathcal{U}_{s}\right) \mathrm{d} s \text { is a } \mathbf{P}^{\delta} \text {-martingale. }
$$

The operator $\mathcal{A}_{\rho^{\delta}}^{\delta}$ is defined by

$$
\mathcal{A}_{\rho^{\delta}}^{\delta}(f)(t, x, u):=\left(u \cdot \nabla_{x} f(x, u)\right)+\left(B_{\delta}\left[x, u ; \rho^{\delta}(t)\right] \cdot \nabla_{u} f(x, u)\right)+\frac{1}{2} \sum_{i, j=1}^{d} a^{(i, j)}(t, x, u) \partial_{u_{i}, u_{j}}^{2} f(x, u) .
$$

The probability measure $\mathbf{P}^{\delta}$ resulting from the particle limit provides a weak solution $\left(X_{t}^{\delta}, \mathcal{U}_{t}^{\delta} ; t \in[0, T]\right)$ to

$$
\left\{\begin{array}{l}
X_{t}^{\delta}=X_{0}+\int_{0}^{t} \mathcal{U}_{s}^{\delta} \mathrm{d} s \\
\mathcal{U}_{t}^{\delta}=\mathcal{U}_{0}+\int_{0}^{t} B_{\delta}\left[X_{s}^{\delta}, \mathcal{U}_{s}^{\delta} ; \rho^{\delta}(s)\right] \mathrm{d} s+\int_{0}^{t} \sigma\left(s, X_{s}^{\delta}, \mathcal{U}_{s}^{\delta}\right) \mathrm{d} W_{s} \\
\rho^{\delta}(t, x, u) \text { is the density of }\left(X_{t}^{\delta}, \mathcal{U}_{t}^{\delta}\right) \text { for all } t \in(0, T]
\end{array}\right.
$$

The proof of Theorem 2.2 proceeds in two main steps: first, for fixed $\delta>0$ and owing to (2.7), we prove a tightness result for the laws $\left\{\overline{\mathbb{P}}^{\delta, N_{p}}:=\mathbb{P} \circ\left(\mu^{\delta, N_{p}}\right)^{-1} ; N_{p}>0\right\}$ ensuring its relative compactness (w.r.t. the weak topology). Next, we show that the limit of every converging subsequence only charges solutions to the martingale problem related to (2.8). We identify the time-marginal densities $\left(\rho^{\delta}(t, \cdot, \cdot) ; t \in[0, T]\right)$ of $\mathbf{P}^{\delta}$ as the unique solution of a mild equation related to $B_{\delta}$. This implies the uniqueness of the solution to (2.8).

It remains to decrease $\delta$ to 0 .

Theorem 2.3. When $\delta$ tends to 0 , the probability measure $\mathbf{P}^{\delta}$ solution to the martingale problem related to (2.8) converges weakly to the unique solution to the martingale problem related to (2.1).

In addition, we have the following:

Theorem 2.4. For all $t \in(0, T]$, the time-marginals $\rho^{\delta}(t)$ converge towards $\rho(t)$ in $L^{1}\left(\mathbb{R}^{2 d}\right)$.

The detailed proofs of the above theorems can be found in [2]. The proof of Theorem 2.4 follows and adapts to our case the general ideas of [33] on the convergence of martingale problems related to strong elliptic diffusion processes.

The propagation of chaos stated in Theorem 2.2 is equivalent to the weak convergence of the random empirical measure $\mu^{\delta, N_{p}}$ to the deterministic measure $\mathbf{P}^{\delta}$ (see [35]). We then obtain the convergence of the conditional expectation estimator by combining the propagation of chaos with the convergences of Theorems 2.3 and 2.4: $\mathbb{P}$-a.s., for a.e. $(t, x) \in(0, T] \times \mathbb{R}^{d}$,

$$
\lim _{\delta \rightarrow 0} \lim _{N_{p} \rightarrow+\infty} \frac{\frac{1}{N_{p}} \sum_{j=1}^{N_{p}} g\left(\mathcal{U}_{t}^{j, \delta, N_{p}}\right) \phi_{\delta}\left(X_{t}^{j, \delta, N_{p}}-x\right)}{\frac{1}{N_{p}} \sum_{j=1}^{N_{p}} \phi_{\delta}\left(X_{t}^{j, \delta, N_{p}}-x\right)+\delta}=\frac{\int_{\mathbb{R}^{d}} g(u) \rho(t, x, u) \mathrm{d} u}{\int_{\mathbb{R}^{d}} \rho(t, x, u) \mathrm{d} u},
$$

for any bounded continuous function $g$. With the notation of Section 1, the right-hand side of the above rewrites as the Eulerian averaged $\langle g(U)\rangle(t, x)$.

\subsection{Confined Lagrangian process with no-permeability condition}

As mentioned in Section 1, the jumps in the dynamics of SDM in (1.1) are meant to ensure the confinement of particles in $\mathcal{D}$, and further to force the boundary conditions (1.2) in the Lagrangian dynamics. Hereafter, 
we detail the confinement of the simplified Lagrangian system (2.1) in the case of the mean no-permeability condition:

$$
\mathbb{E}\left[\left(\mathcal{U}_{t} \cdot n_{\mathcal{D}}\left(X_{t}\right)\right) / X_{t}=x\right]=0 \text { for } t \in(0, T], x \in \partial \mathcal{D} .
$$

Here, $\mathcal{D}$ is assumed to be a smooth open subset of $\mathbb{R}^{d}$ (not necessary bounded), and $n_{\mathcal{D}}$ denotes the outward unit vector of $\mathcal{D}$. According to (1.12), the condition (2.10) reads as nil flux for the outward Eulerian velocity: $\left(\langle U\rangle(t, x) \cdot n_{\mathcal{D}}(x)\right)=0$. This boundary condition provides an example of limit condition to the averaged Euler equation (1.14).

Considering an appropriate notion of trace $\gamma(\rho)(t, x, u)$ of the density $\rho(t, x, u)$ of $\left(X_{t}, \mathcal{U}_{t}\right)$, the condition $(2.10)$ writes:

$$
\frac{\int_{\mathbb{R}^{d}}\left(u \cdot n_{\mathcal{D}}(x)\right) \gamma(\rho)(t, x, u) \mathrm{d} u}{\int_{\mathbb{R}^{d}} \gamma(\rho)(t, x, u) \mathrm{d} u}=0 \text { for } t \in(0, T], x \in \partial \mathcal{D} .
$$

Moreover, when $\mathcal{D}=\mathbb{R}^{d}$ we observe that the time-marginal densities $(\rho(t, \cdot, \cdot), t \in(0, T])$ of the solution to $(2.1)$ satisfies the following Fokker-Planck equation in the sense of distributions

$$
\partial_{t} \rho(t, x, u)+\mathcal{A}_{\rho}^{*}(\rho)(t, x, u)=0 \text { for }(t, x, u) \in(0, T) \times \mathcal{D} \times \mathbb{R}^{d},
$$

where $\mathcal{A}_{\rho}^{*}$ denotes the formal adjoint of $\mathcal{A}_{\rho}$ defined in (2.3). Here $\mathcal{D} \neq \mathbb{R}^{d}$ and the notion of trace $\gamma(\rho)$ shall be defined through the following Green formula: for all $t \in[0, T]$, for all functions $\psi \in \mathcal{C}^{\infty}\left([0, t] \times \overline{\mathcal{D}} \times \mathbb{R}^{d}\right)$,

$$
\begin{aligned}
\int_{(0, t) \times \mathcal{D} \times \mathbb{R}^{d}} \rho(s, x, u)\left(\partial_{s} \psi(s, x, u)+\mathcal{A}_{\rho}(\psi)(s, x, u)\right) \mathrm{d} s \mathrm{~d} x \mathrm{~d} u=\int_{\mathcal{D} \times \mathbb{R}^{d}} \rho(t, x, u) \psi(t, x, u) \mathrm{d} x \mathrm{~d} u \\
-\int_{\mathcal{D} \times \mathbb{R}^{d}} \psi(0, x, u) \mu_{0}(\mathrm{~d} x \mathrm{~d} u)+\int_{(0, t) \times \partial \mathcal{D} \times \mathbb{R}^{d}}\left(u \cdot n_{\mathcal{D}}(x)\right) \gamma(\rho)(s, x, u) \psi(s, x, u) \mathrm{d} \lambda_{\Sigma}(s, x, u),
\end{aligned}
$$

where $\mathrm{d} \lambda_{\Sigma}$ denotes the product measure $\mathrm{d} s \mathrm{~d} \sigma_{\mathcal{D}}(x) \mathrm{d} u, \mathrm{~d} \sigma_{\mathcal{D}}$ being the surface measure of $\partial \mathcal{D}$. We shall also impose that

$$
\int_{\mathbb{R}^{d}}\left|\left(u \cdot n_{\mathcal{D}}(x)\right) \gamma(\rho)(t, x, v)\right| \mathrm{d} v\left\langle+\infty, \text { and } \int_{\mathbb{R}^{d}}|\gamma(\rho)(t, x, v)| \mathrm{d} v\right\rangle 0,
$$

so that the left member of (2.11) is well defined.

Now we observe that a sufficient condition for the trace $\gamma(\rho)$ to satisfy the mean no-permeability condition $(2.10)$ is

$$
\gamma(\rho)(t, x, u)=\gamma(\rho)\left(t, x, u-2\left(u \cdot n_{\mathcal{D}}(x)\right) n_{\mathcal{D}}(x)\right) \text { for }(t, x, u) \in(0, T) \times \partial \mathcal{D} \times \mathbb{R}^{d} .
$$

This (spatial) boundary condition, called specular reflection boundary condition, belongs to the range of Maxwell's boundary conditions introduced for kinetic equations such as Boltzmann and Vlasov-Fokker-Planck equations. These conditions model the reflexion/diffusion processes of gas particles in the presence of solid boundaries (see e.g. [5]).

Following this preliminary idea, we now discuss the well-posedness of the related simplified Lagrangian system:

$$
\left\{\begin{array}{l}
X_{t}=X_{0}+\int_{0}^{t} \mathcal{U}_{s} \mathrm{~d} s \\
\mathcal{U}_{t}=\mathcal{U}_{0}+\int_{0}^{t} B\left[X_{s}, \mathcal{U}_{s} ; \rho(s)\right] \mathrm{d} s+\int_{0}^{t} \sigma\left(s, X_{s}, \mathcal{U}_{s}\right) \mathrm{d} W_{s}+K_{t}, \\
\rho(t, x, u) \text { is the density distribution of }\left(X_{t}, \mathcal{U}_{t}\right) \text { for all } t \in(0, T]
\end{array}\right.
$$


where the confining process $K$ is defined by

$$
K_{t}=-2 \sum_{0<s \leq t}\left(\mathcal{U}_{s^{-}} \cdot n_{\mathcal{D}}\left(X_{s}\right)\right) n_{\mathcal{D}}\left(X_{s}\right) \mathbb{1}_{\left\{X_{s} \in \partial \mathcal{D}\right\}}
$$

The kernel $B$ is unchanged, defined as in (2.2).

The existence of a solution to (2.15) is subjected to the existence and the growth to infinity of the sequence of hitting times $\left\{\tau_{n}\right\}_{n \in \mathbb{N}}$ defined by

$$
\left\{\begin{array}{l}
\tau_{0}=\inf \left\{t \geq 0 \text { s.t. } X_{t} \in \partial \mathcal{D}\right\} \\
\tau_{n}=\inf \left\{t>\tau_{n-1} \text { s.t. } X_{t} \in \partial \mathcal{D}\right\}, \text { for } n \geq 1
\end{array}\right.
$$

The martingale problem related to $(2.15)-(2.17)$ is formulated as follows: consider $\Omega:=\mathcal{C}\left([0, T] ; \mathbb{R}^{d}\right) \times$ $\mathbb{D}\left([0, T] ; \mathbb{R}^{d}\right) \times \mathbb{D}\left([0, T] ; \mathbb{R}^{d}\right)$ (where $\mathbb{D}\left([0, T] ; \mathbb{R}^{d}\right)$ is the space of càdlàg functions defined on $[0, T]$, and taking values in $\left.\mathbb{R}^{d}\right)$, and $\left(X_{t}, \mathcal{U}_{t}, K_{t} ; t \in[0, T]\right)$ the related canonical processes. A probability measure $\mathbf{P}$ is said to be a solution to the martingale problem related to $(2.15)-(2.17)$ if the following conditions hold:

$(P 1) \mathbf{P} \circ\left(X_{0}, \mathcal{U}_{0}\right)^{-1}=\mu_{0}$, and, for all $t \in(0, T], \mathbf{P}_{t}:=\mathbf{P} \circ\left(X_{t}, \mathcal{U}_{t}\right)^{-1}$ admits a positive density $\rho(t, x, u)$ w.r.t. Lebesgue measure.

$(P 2)$ For all $f \in \mathcal{C}_{b}^{2}\left(\mathbb{R}^{2 d}\right)$,

$$
M_{t}:=f\left(X_{t}, \mathcal{U}_{t}-K_{t}\right)-f\left(X_{0}, \mathcal{U}_{0}\right)-\int_{0}^{t} \mathcal{A}_{\rho}(f)\left(s, X_{s}, \mathcal{U}_{s}-K_{s}\right) \text { d } s \text { is a } \mathbf{P} \text {-martingale }
$$

where $\mathcal{A}_{\rho}$ is defined as in $(2.3)$.

(P3) P-a.s. $t \mapsto X_{t}$ hits at most countably many times the boundary $\partial \mathcal{D}$, and $\left(K_{t} ; t \in[0, T]\right)$ is given by $(2.16)$.

Consider a weak solution (in the sense of the martingale problem) to (2.15)-(2.17). Itô's formula applied to $(X, \mathcal{U})$, we show that, for all $t>0, f \in \mathcal{C}_{c}\left([0, t] \times \partial \mathcal{D} \times \mathbb{R}^{d}\right)$,

$$
\begin{aligned}
\sum_{n \in \mathbb{N}} & \mathbb{E}_{\mathbb{P}}\left[\left(f\left(\tau_{n}, X_{\tau_{n}}, \mathcal{U}_{\tau_{n}}\right)-f\left(\tau_{n}, X_{\tau_{n}}, \mathcal{U}_{\tau_{n}^{-}}\right)\right) \mathbb{1}_{\left\{\tau_{n} \leq t\right\}}\right] \\
= & \int_{(0, t) \times \partial \mathcal{D} \times \mathbb{R}^{d}}\left(u \cdot n_{\mathcal{D}}(x)\right) \gamma(\rho)(s, x, u) f(s, x, u) \mathrm{d} \lambda_{\Sigma}(s, x, u) .
\end{aligned}
$$

Hence, $\left(u \cdot n_{\mathcal{D}}(x)\right) \gamma(\rho)(t, x, u)$ is the density of $\sum_{n \in \mathbb{N}} \mathbb{P} \circ\left(\tau_{n}, X_{\tau_{n}}, \mathcal{U}_{\tau_{n}}\right)^{-1}$ with respect to the measure $\mathrm{d} \lambda_{\Sigma}$. From (2.18), one can deduce the positivity and integrability properties (2.13) of the trace $\gamma(\rho)$. Moreover, the density and trace $(\rho, \gamma(\rho))$ satisfy the Green formula (2.12). Then, a straightforward computation shows that, as long as the specular boundary condition is fulfilled, the solution to (2.15)-(2.17) satisfies the constraint (2.10).

The main difficulty remains the existence of a solution to (2.15)-(2.17).

In Section 2.2.1, we take profit of the particular choice of an hyperplane for $\mathcal{D}$ to construct a solution to (2.15)(2.17). In this situation, we are able to extend the results of well-posedness and particle approximation developed in the previous section.

In Section 2.2.2, we consider a more general domain. A first well-posedness result for the time-marginals related to (2.15) is then obtained. 


\subsubsection{Confinement in an hyperplane}

We consider the domain $\mathcal{D}=\mathbb{R}^{d-1} \times(0,+\infty)$. The confinement procedure in (2.15) only concerns the $d^{\text {th }}$ component of the velocity. Thus one can focus on the study of the solution to

$$
\left\{\begin{array}{l}
X_{t}=X_{0}+\int_{0}^{t} \mathcal{U}_{s} \mathrm{~d} s \\
\mathcal{U}_{t}=\mathcal{U}_{0}+\int_{0}^{t} B\left[X_{s}, \mathcal{U}_{s} ; \rho(s)\right] \mathrm{d} s+W_{t}-2 \sum_{0<s \leq t} \mathcal{U}_{s^{-}} \mathbb{1}_{\left\{X_{s}=0\right\}}
\end{array}\right.
$$

We preliminary investigate the properties of the solution to $(2.19)$ when $b=0$. In this situation, we have an explicit solution thanks to the following simple construction (see $[16])$ : considering $\left(Y_{t}^{x_{0}, u_{0}}, V_{t}^{u_{0}} ; t \in[0, T]\right.$ ) solution to

$$
\left\{\begin{array}{l}
Y_{t}^{x_{0}, u_{0}}=x_{0}+\int_{0}^{t} V_{s}^{u_{0}} \mathrm{~d} s, \\
V_{t}^{u_{0}}=u_{0}+W_{t}
\end{array}\right.
$$

we construct $\left(X_{t}^{x_{0}, u_{0}}, \mathcal{U}_{t}^{x_{0}, u_{0}} ; t \in[0, T]\right)$ as the trajectorial transformation of $\left(Y_{t}^{x_{0}, u_{0}}, V_{t}^{x_{0}, u_{0}} ; t \in[0, T]\right)$ reflecting the velocity each time the particle position reaches zero. We proceed by defining

$$
\begin{aligned}
& X_{t}^{x_{0}, u_{0}}=\left|Y_{t}^{x_{0}, u_{0}}\right|, \\
& \mathcal{U}_{t}^{x_{0}, u_{0}}=\mathcal{S}_{t}^{x_{0}, u_{0}} V_{t}^{u_{0}} \text { for all } t \in(0, T],
\end{aligned}
$$

where $\mathcal{S}_{t}^{x_{0}, u_{0}}:=\operatorname{sign}\left(Y_{t}^{x_{0}, u_{0}}\right)^{+}$denotes the càdlàg version of $\operatorname{sign}\left(Y_{t}^{x_{0}, u_{0}}\right)$.

This construction requires to estimate the excursion times at 0 of the primitive of the Brownian motion. In $[18,20]$, the authors prove that, starting from $x_{0}>0$, the $1 D$-Brownian's primitive has only countably many excursions from 0 , they also explicit the law of the related hitting sequence.

As a result, the density $\rho^{c}\left(0, x_{0}, u_{0} ; t, x, u\right)$ of $\left(X_{t}^{x_{0}, u_{0}}, \mathcal{U}_{t}^{x_{0}, u_{0}}\right)$ can be written in terms of the distribution $\rho\left(0, x_{0}, u_{0} ; t, x, u\right)$ of $\left(Y_{t}^{x_{0}, u_{0}}, V_{t}^{x_{0}, u_{0}}\right)$ :

$$
\rho^{c}\left(0, x_{0}, u_{0} ; t, x, u\right)=\left(\rho\left(0, x_{0}, u_{0} ; t, x, u\right)+\rho\left(0, x_{0}, u_{0} ; t,-x,-u\right)\right) \mathbb{1}_{\{x \geq 0\}} .
$$

As $\rho^{c}\left(0, x_{0}, u_{0} ; t, x, u\right)$ is explicitly known, we further check that the properties stated in Lemma 2.1 hold, and thus ensure the existence of the particle system

$$
\left\{\begin{array}{l}
X_{t}^{i, \delta, N_{p}}=X_{0}^{i}+\int_{0}^{t} \mathcal{U}_{s}^{i, \delta, N_{p}} \mathrm{~d} s \\
\mathcal{U}_{t}^{i, \delta, N_{p}}=\mathcal{U}_{0}^{i}+\int_{0}^{t} \frac{\sum_{j=1}^{N_{p}} b\left(\mathcal{U}_{s}^{j, \delta, N_{p}}, \mathcal{U}_{s}^{i, \delta, N_{p}}\right) \phi_{\delta}\left(X_{s}^{i, \delta, N_{p}}-X_{s}^{j, \delta, N_{p}}\right)}{\sum_{j=1}^{N_{p}}\left(\phi_{\delta}\left(X_{s}^{i, \delta, N_{p}}-X_{s}^{j, \delta, N_{p}}\right)+\delta\right)} \mathrm{d} s+W_{t}^{i}-2 \sum_{0<s \leq t} \mathcal{U}_{s}^{i, \delta, N_{p}} \mathbb{1}_{\left\{X_{s}^{i, \delta, N_{p}}=0\right\}}
\end{array}\right.
$$

Replicating the arguments introduced in Section 2.2, we are able to prove the following theorem (see [16]).

Theorem 2.5. Suppose $\mathcal{D}=\mathbb{R}^{d-1} \times(0,+\infty)$, and assume that $\left(X_{0}, \mathcal{U}_{0}\right)$ are distributed according to a probability measure $\mu_{0}$ satisfying (2.7), and having no support on $\{0\} \times \mathbb{R}$. Then, as $N_{p}$ grows to $+\infty$, and $\delta$ tends to 0 , the empirical measure $\mu^{\delta, N_{p}}:=1 / N_{p} \sum_{j=1}^{N_{p}} \delta_{\left\{X^{\left.j, \delta, N_{p}, \mathcal{U}^{j, \delta, N_{p}}\right\}}\right.}$ of the particle system (2.22) converges in the weak sense to the unique solution $\mathbf{P}$ to the martingale problem related to (2.19), (2.17).

Moreover, the time marginal density $\rho(t, x, u)$ of $\mathbf{P}$ admits a trace and satisfies the specular condition (2.14). The corresponding processes $(X, \mathcal{U})$ fulfill the mean no-permeability condition $(2.10)$. 
Remark 2.6. In the particular case where the one-dimensional model (2.19) endows a "linear" drift $B(t, x, u)$, the explicit construction (2.20) and the corresponding relation (2.21) remain valid. Given the drift $B(t, x, u)$, the associated free process $\left(Y^{x_{0}, u_{0}}, V^{x_{0}, u_{0}}\right)$ to consider in $(2.21)$ is the solution to

$$
\left\{\begin{array}{l}
Y_{t}^{x_{0}, u_{0}}=x_{0}+\int_{0}^{t} V_{s}^{x_{0}, u_{0}} \mathrm{~d} s \\
V_{t}^{x_{0}, u_{0}}=u_{0}+\int_{0}^{t} \widetilde{B}\left(s, Y_{s}^{x_{0}, u_{0}}, V_{s}^{x_{0}, u_{0}}\right) \mathrm{d} s+W_{t},
\end{array}\right.
$$

where $\widetilde{B}$ is defined by

$$
(t, x, u) \mapsto \widetilde{B}(t, x, u)=\operatorname{sign}(x) B(t,|x|, \operatorname{sign}(x) u) .
$$

\subsubsection{A PDE approach}

Compared to the case of the hyperplane, the well-posedness of the confined Lagrangian models (2.15) in a smooth bounded domain, submitted to (2.10) is far more difficult to handle. A first attempt on this problem, considered in [16], is to look at the McKean-Vlasov-Fokker-Planck equation describing the time-marginal densities related to a confined Lagrangian system of the form (2.15). As we have seen, this equation may write:

$$
\left\{\begin{array}{l}
\partial_{t} \rho+\left(u \cdot \nabla_{x} \rho\right)+\left(B[; \rho] \cdot \nabla_{u} \rho\right)-\frac{\sigma^{2}}{2} \triangle_{u} \rho=0 \text { on }(0, T) \times \mathcal{D} \times \mathbb{R}^{d} \\
\rho(0, x, u)=\rho_{0}(x, u) \text { in } \mathcal{D} \times \mathbb{R}^{d} \\
\gamma(\rho)(t, x, u)=\gamma(\rho)\left(t, x, u-2\left(u \cdot n_{\mathcal{D}}(x)\right) n_{\mathcal{D}}(x)\right) \text { on }(0, T] \times \partial \mathcal{D} \times \mathbb{R}^{d}
\end{array}\right.
$$

where $\rho_{0}$ stands for the initial distribution. Here, the coefficient $B$ is a mapping from $(0, T) \times \mathcal{D} \times L^{1}((0, T) \times \mathcal{D})$ to $\mathbb{R}^{d}$ defined as

$$
B[t, x ; \gamma]=\left\{\begin{array}{l}
\frac{\int_{\mathbb{R}^{d}} b(v) \gamma(t, x, v) \mathrm{d} v}{\int_{\mathbb{R}^{d}} \gamma(t, x, v) \mathrm{d} v} \text { if } \int_{\mathbb{R}^{d}} \gamma(t, x, v) \mathrm{d} v \neq 0, \\
0 \text { elsewhere, }
\end{array}\right.
$$

where $b: \mathbb{R}^{d} \rightarrow \mathbb{R}^{d}$ is bounded and Borel measurable. Again, $\gamma(\rho)$ denotes the trace of $\rho$, defined through the Green formula to (2.12), and has to satisfy the conditions (2.13).

Equation (2.24) belongs to the class of linear Vlasov-Fokker-Planck equations. Well-posedness of a nonnegative weak solution (with $\rho \in L^{2}\left((0, T) \times \mathcal{D} ; H^{2}\left(\mathbb{R}^{d}\right)\right.$ ) where $H^{2}$ denotes the usual $L^{2}$-Sobolev space), and theorem on the existence of trace have been proved for linear version of $(2.24)$ (see e.g. [4,8,9]). We prove an energy inequality which provides an uniqueness result to (2.24) in the set $\mathbf{V}$ of solutions:

$$
\mathbf{V}:=\left\{\rho \in L_{w}^{2}\left((0, T) \times \mathcal{D} ; H_{w}^{2}\left(\mathbb{R}^{d}\right)\right) ; \sup _{(t, x)} \int_{\mathbb{R}^{d}} w(u)|\rho(t, x, u)|^{2} \mathrm{~d} u<+\infty \text { and } \int_{\mathbb{R}^{d}} \rho(t, x, u) \mathrm{d} u>0\right\} .
$$

Here $H_{w}^{2}$ denotes the weighted Sobolev space endowing the weight $w(u)=\left(1+|u|^{2}\right)^{\frac{\alpha}{2}}$ for $\alpha>d$.

In order to handle the singularity in the nonlinear coefficient $B[\cdot, \cdot ; \rho]$, a suitable tool is provided by the construction of upper and lower Gaussian bounds resulting from the existence of Maxwellian super- and subsolutions for equation (2.24). The considered Maxwellian functions have the following form:

$$
M(t, u)=\exp (-a t)(m(t, u))^{\beta}
$$


where $\beta>0$, and $m(t, u)$ is the solution to the heat equation:

$$
\left\{\begin{array}{l}
\partial_{t} m-\frac{\sigma^{2}}{2} \triangle_{u} m=0 \text { on }(0, T] \times \mathbb{R}^{d}, \\
m(0, u)=m_{0}(u) .
\end{array}\right.
$$

Assuming the following a priori bounds on $\rho_{0}$,

$$
\underline{m}_{0}(u) \leq \rho_{0}(x, u) \leq \bar{m}_{0}(u), \quad(x, u) \in \mathcal{D} \times \mathbb{R}^{d},
$$

we choose appropriately the parameters $\beta$ and $a$ in terms of $\sigma$ and $\|b\|_{\infty}$ and by means of comparison arguments, similar lower and upper bounds $\underline{M}, \bar{M}$ are found for the solution $\rho$ to equation $(2.24)$.

Using a fixed point method, together with compactness and contraction arguments, one obtains the wellposedness of a weak solution to the nonlinear equation (2.24):

Theorem 2.7. Assume (2.25) with $\underline{m}_{0}>0$ and $\bar{m}_{0} \in L_{w}^{2}$. There exists a unique weak solution $\rho$ to (2.24), and a couple of Maxwellian functions $(\underline{M}, \bar{M})$ depending on $\underline{m}_{0}, \bar{m}_{0}, \sigma$, and $\|b\|_{\infty}$ such that

$$
\begin{aligned}
& \underline{M}(t, u) \leq \rho \leq \bar{M}(t, u) \text { on }(0, T) \times \mathcal{D} \times \mathbb{R}^{d}, \\
& \underline{M}(t, u) \leq \gamma(\rho) \leq \bar{M}(t, u) \text { on }(0, T) \times \partial \mathcal{D} \times \mathbb{R}^{d} .
\end{aligned}
$$

An important corollary of Theorem 2.7 is the uniqueness of the solution to (2.15). The construction of the Lagrangian process solution to (2.15) is a work under progress.

The results outlined in this section deal with well-posedness and particle approximation issues for simplified versions of stochastic Lagrangian model for downscaling methods. Their extensions to SDM and other stochastic Lagrangian models (see $[27,28]$ ) call for deeper developments, as they feature unbounded drift terms (as in (1.1) and (1.3)), and diffusion coefficients involving non-linearity of conditional type which is a hard topic in the general framework of non-linear SDEs.

\section{NumericAl DESCRIPTION OF SDM}

We present in this section the numerical discretization of SDM. We apply a splitting scheme in which the pressure gradient effects (the constant mass and mean free divergence constraints (1.5) and (1.6)) are taken into account thanks to a projection step (see details in Sect. 3.2 below).

The stochastic differential equations that we consider here are

$$
\left\{\begin{aligned}
X_{t}= & X_{0}+\int_{0}^{t} \mathcal{U}_{s} \mathrm{~d} s \\
\mathcal{U}_{t} & =\mathcal{U}_{0}-\left(\frac{1}{2}+\frac{3}{4} C_{0}\right) \frac{C_{\varepsilon}}{\ell_{m}} \int_{0}^{t}\left(k_{L}\left(s, X_{s}\right)\right)^{1 / 2}\left(\mathcal{U}_{s}-\langle U\rangle\left(s, X_{s}\right)\right) \mathrm{d} s+\sqrt{\frac{C_{0} C_{\varepsilon}}{\ell_{m}}} \int_{0}^{t}\left(k_{L}\left(s, X_{s}\right)\right)^{3 / 4} \mathrm{~d} W_{s} \\
& +2 \sum_{0<s \leq t}\left(U_{\text {ext }}\left(s, X_{s}\right)-\mathcal{U}_{s^{-}}\right) \mathbb{1}_{\left\{X_{s} \in \partial \mathcal{D}\right\}}
\end{aligned}\right.
$$

In comparison with equation (1.1), the reader will notice that we omit the pressure gradient term in equation (3.1). We consider a particle discretization of the Lagrangian equation (3.1), using the Particle in Cell method for the computation of Eulerian quantities such as $\langle U\rangle(t, x)$. As shown in Section 2, an interacting particle system associated to (3.1) allows to approximate its Eulerian quantities. In particular, using the notations 
of Section 2, for any bounded smooth function $Q$, the quantity

$$
\frac{\frac{1}{N_{p}} \sum_{j=1}^{N_{p}} Q\left(\mathcal{U}_{t}^{j, \delta, N_{p}}\right) \phi_{\delta}\left(x-X_{t}^{j, \delta, N_{p}}\right)}{\frac{1}{N_{p}} \sum_{j=1}^{N_{p}} \phi_{\delta}\left(x-X_{t}^{j, \delta, N_{p}}\right)+\delta}
$$

is a converging estimator of $\mathbb{E}\left(Q\left(\mathcal{U}_{t}\right) / X_{t}=x\right)$, as $N_{p}$ goes to $\infty$ and $\delta$ goes to 0 (see (2.9)). In the class of such Nadaraya-Watson estimators, we are interested in the Nearest Grid Point estimator (see Sect. 3.1 below), particularly well adapted to SDM.

As far as the time discretization is concerned, we use an explicit Euler method, for which an exponential scheme is considered in order to ensure long time stability (see Sect. 3.2). Each time iteration consists in a sequence of three sub-steps: the first step updates the particle properties without taking the pressure term into account.

The second step only concerns the particles hitting the boundary. It consists in a reflection on both velocity and position. The jump applied on the velocity is determined in terms of the Dirichlet condition (1.2). The reflection carried out in Section 3.2 is original and allows to transfer the Eulerian boundary conditions to the local Lagrangian model.

In order to avoid local numerical instabilities created by a stiff treatment of the particles at the boundary, we introduce a regularization of (3.1), for which the first equation is replaced by

$$
\left\{\begin{array}{l}
X_{t}=X_{0}+\int_{0}^{t} \mathcal{V}_{s} \mathrm{~d} s, \\
\mathcal{V}_{t}=\mathcal{U}_{t}-h_{\delta}\left(X_{t}\right) U_{\text {ext }}\left(t, X_{t}\right),
\end{array}\right.
$$

where $h_{\delta}$ is the indicator function of $\mathcal{B}_{\delta}=\{x \in \mathcal{D} ;|x-\partial \mathcal{D}|<\delta\}$. The process $\left(\mathcal{V}_{t}, t \in[0, T]\right)$ above represents the Lagrangian velocity field (see the first equation of (3.3)), which is shifted by $-U_{\text {ext }}$ (see second equation) for any particle that moves inside the boundary neighborhood $\mathcal{B}_{\delta}$.

Finally, the third step projects the unknowns onto the constraint space (constant mass density and divergencefree velocity).

\subsection{The Particle in Cell method}

We briefly present in this section the Particle in Cell (PIC) method, that is very well described and analysed in [29]. Given a Lagrangian field, the PIC method aims at computing local averages of the particle properties in order to obtain the corresponding Eulerian variables, such as the three-dimensional velocity.

From a numerical point of view, it is classical to carry out such approximations by using the Nearest Grid Point (NGP) method. In this way, we drop $N_{p}$ fluid particles in the domain $\mathcal{D}$ and mesh $\mathcal{D}$ into $N_{c}$ disjoint cells $\left(\mathcal{C}_{i}, i=1, \ldots, N_{c}\right)$ so that $\mathcal{D}=\cup_{i=1}^{N_{c}} C_{i}$. Let us denote by $\left(X_{t}^{k, N_{p}}, \mathcal{U}_{t}^{k, N_{p}}\right)$ the position and velocity at time $t$, of the $k^{\text {th }}$ particle among $N_{p}$. For all $x \in \mathcal{D}$, we denote by $i_{x}$ the cell number such that $x \in \mathcal{C}_{i_{x}}$, and $\mathbb{1}_{\left\{\cdot \in \mathcal{C}_{i_{x}}\right\}}$ is the indicator function of $\mathcal{C}_{i_{x}}$. Then the quantity $\mathbb{E}\left(Q\left(\mathcal{U}_{t}\right) / X_{t}=x\right)$, involved in (3.1) with ad hoc expressions of $Q$, is approximated by :

$$
\begin{aligned}
\langle Q(U)\rangle(t, x) & =\mathbb{E}\left(Q\left(\mathcal{U}_{t}\right) / X_{t}=x\right) \\
& \simeq\left\{\begin{array}{l}
\sum_{k=1}^{N_{p}} Q\left(\mathcal{U}_{t}^{k, N_{p}}\right) \mathbb{1}_{\left\{X_{t}^{k, N_{p}} \in \mathcal{C}_{i_{x}}\right\}} \\
\#\left\{X_{t}^{k, N_{p}} \in \mathcal{C}_{i_{x}}, k=1, \ldots, N_{p}\right\} \\
0 \text { elsewhere, }
\end{array} \text { if } \#\left\{X_{t}^{j, N_{p}} \in \mathcal{C}_{i_{x}}, j=1, \ldots, N_{p}\right\} \neq 0,\right.
\end{aligned}
$$


which is closed to (3.2) if we define the function $\phi_{\delta}$ thanks to the above indicator functions. In the SDM approach (3.1), $x$ is always the center of a cell $C_{i}$. Moreover, due to the uniform distribution of the particles as expressed in (1.5), the estimator (3.4) is reduced to

$$
\mathbb{E}\left(Q\left(\mathcal{U}_{t}\right) / X_{t}=x\right) \simeq \frac{1}{N_{p c}} \sum_{k=1}^{N_{p}} Q\left(\mathcal{U}_{t}^{k, N_{p}}\right) \mathbb{1}_{\left\{X_{t}^{k, N_{p}} \in \mathcal{C}_{i_{x}}\right\}}
$$

where $N_{p c}$ denotes the constant number of particles per cell.

Note that it is possible to replace the NGP method by higher order methods (based on smoother splines) that take into account more particles in the cell neighborhood. These methods increase the numerical complexity and require a particular treatment for the computation of Eulerian quantities in the boundary cells.

By then, any statistics on the Eulerian velocity $U$ (mean components, variances, turbulent kinetic energy $k$ ) is evaluated in a cell $\mathcal{C}_{i}$ by an average over the $N_{p c}$ particles present in $\mathcal{C}_{i}$.

The convergence speed of the first and second moment estimators should behave (at least asymptotically) as a Monte Carlo method (independent sampling). Thus, we expect the $95 \%$ confidence interval for the mean wind components to be driven by the local turbulent variance $\bar{\sigma}^{2}=\left(\left\langle u^{2}\right\rangle,\left\langle v^{2}\right\rangle,\left\langle w^{2}\right\rangle\right)$. The error between the numerical velocity $\langle U\rangle_{\mathrm{SDM}}$ and the reference solution $\langle U\rangle$ in each cell $\mathcal{C}_{i}$ may be quantified with the help of the law of large numbers and the central limit theorem as:

$$
\mathbb{P}\left(\langle U\rangle-\langle U\rangle_{\mathrm{SDM}} \in\left[-\frac{2 \bar{\sigma}}{\sqrt{N_{p c}}}, \frac{2 \bar{\sigma}}{\sqrt{N_{p c}}}\right]\right) \geq 95 \%
$$

\subsection{The numerical time-scheme}

We present hereafter the numerical discretization of (3.1). For robustness considerations (see [23]), we consider the exponential version of the explicit Euler scheme for the prediction step (Step 1). We propose in Step 2 an original method to confine particles in $\mathcal{D}$ according to the following downscaling principle: the inferred Eulerian velocity field satisfies the Dirichlet condition (1.2).

At time $t_{n-1}=(n-1) \Delta t$, the $N_{p}$ Lagrangian variables $\left(X_{n-1}^{k}, \mathcal{U}_{n-1}^{k}\right):=\left(X_{n-1}^{k, N_{p}}, \mathcal{U}_{n-1}^{k, N_{p}}\right)$ are known, as well as the statistics $k_{n-1}$ and $\left\langle U_{n-1}\right\rangle$ in each cell $\mathcal{C}$ of the partition of $\mathcal{D}=\cup_{i=1}^{N_{c}} \mathcal{C}_{i}$. At time $t_{n}$, for each particle $k$ :

Step 1. Prediction: We compute the following quantities:

- The particle velocity $\mathcal{V}_{n-1}^{k}=\mathcal{U}_{n-1}^{k}-U_{\text {ext }}\left(t_{n-1}, X_{n-1}^{k}\right) h_{\delta}\left(X_{n-1}^{k}\right)$;

- The particle position $\widetilde{X}_{n}^{k}=X_{n-1}^{k}+\Delta t \mathcal{V}_{n-1}^{k}$;

- The velocity $\widetilde{\mathcal{U}}_{n}^{k}$ is calculated applying an exponential scheme to the equation

$$
\mathrm{d} \widetilde{\mathcal{U}}_{t}^{k}=-C_{1} k_{n-1}^{1 / 2}\left(\widetilde{\mathcal{U}}_{t}^{k}-\left\langle U_{n-1}\right\rangle\right) \mathrm{d} t+C_{2} k_{n-1}^{3 / 4} \mathrm{~d} W_{t}, \quad t \in\left[t_{n-1}, t_{n}\right]
$$

where $\left\langle U_{n-1}\right\rangle, k_{n-1}$ and $\varepsilon_{n-1}$ are evaluated in the cell containing $X_{n-1}^{k}$. If $\widetilde{X}_{n}^{k} \in \mathcal{D}$, then set $X_{n}^{k}=\widetilde{X}_{n}^{k}$ and $\mathcal{U}_{n}^{k}=\widetilde{\mathcal{U}}_{n}^{k}$.

Step 2. Reflection: When $\tilde{X}_{n}^{k} \notin \mathcal{D}$; let $t_{\text {out }}$ be the boundary hitting time after $t_{n-1}$, and $x_{\text {out }}=X_{n-1}^{k}+\left(t_{\text {out }}-\right.$ $\left.t_{n-1}\right) \mathcal{V}_{n-1}^{k}$ be the hitting position, then the reflected position is set to

$$
X_{n}^{k}=x_{\text {out }}-\left(t_{n}-t_{\text {out }}\right) \mathcal{V}_{n-1}^{k}
$$

In concern of the velocity, we simulate equation (3.7) between $t_{n-1}$ and $t_{\text {out }}$ with an exponential scheme to obtain the velocity $\mathcal{U}_{t_{\text {out }}{ }^{k}}^{k}$. Then, in order to match the boundary conditions, we impose a jump 
on the velocity at $t=t_{\text {out }}$ :

$$
\mathcal{U}_{t_{\text {out }}+}^{k}=2 U_{\text {ext }}\left(t_{n-1}, x_{\text {out }}\right)-\mathcal{U}_{t_{\text {out }}{ }^{-}}^{k}
$$

We finally compute $\mathcal{U}_{n}^{k}$ thanks to the simulation of equation (3.7) between $t_{\text {out }}$ and $t_{n}$.

Remark 3.1. In a three-dimensional domain, it may happen that $X_{n}^{k}$ written in (3.8) remains outside the computational domain after the reflection, for instance in the neighborhood of the corners. In this case, new hitting coordinates $x_{\text {out }_{2}}$ are computed, and the new particle position is set to

$$
X_{n}^{k}=x_{\mathrm{out}_{2}}+\gamma
$$

where $\gamma$ is a small vector pushing $x_{\text {out }_{2}}$ inside $\mathcal{D}$. The new velocity $\mathcal{U}_{n}^{k}$ is unchanged.

Step 3. Conservation constraints: Once the $N_{p}$ particles are advanced at time $t_{n}$,

- Move the particles such that there is exactly the same number $N_{p c}$ of particles per cell to fulfill the mass density constraint. The interested reader is referred to [6,7] for further details.

- Compute the new Eulerian quantities $\left\langle\widetilde{U}_{n}\right\rangle$, and project the new Eulerian velocity field on the divergence free space. This may be done thanks to the classical resolution of a Poisson equation for the pressure, with homogeneous Neumann boundary conditions, see [14].

Remark 3.2. The projection method that is classically used to set the divergence of a given (Eulerian) velocity field to zero can not guarantee the boundary conditions to fully match the Dirichlet conditions. Indeed, provided a predicted velocity field $\widetilde{V}$, the projection method consists in solving the following Poisson problem

$$
\left\{\begin{array}{l}
-\Delta \Phi=\frac{1}{\Delta t} \operatorname{div} \tilde{V} \\
\frac{\partial \Phi}{\partial n}=0
\end{array}\right.
$$

Then, the corrected velocity $V$ is computed thanks to

$$
V=\widetilde{V}-\Delta t \nabla \Phi
$$

In our case, $\widetilde{V}$ is $\left\langle\widetilde{U}_{n}\right\rangle$ and $V$ is $\left\langle U_{n}\right\rangle$. After computing $\Phi$ as in (3.10), we actually modify the Lagrangian quantities

$$
\mathcal{U}_{n}^{k}=\widetilde{\mathcal{U}}_{n}^{k}-\Delta t \nabla \Phi\left(X_{n}^{k}\right), \quad k=1, \ldots N_{p},
$$

and finally, it is easy to check that our corrected Eulerian velocity $\left\langle U_{n}\right\rangle$ is such that:

$$
\begin{aligned}
& \nabla_{x} \cdot\left\langle U_{n}\right\rangle=0, \\
& \left\langle U_{n}\right\rangle \cdot \vec{n}=U_{\mathrm{ext}} \cdot \vec{n}, \\
& \text { and possibly }\left\langle U_{n}\right\rangle \cdot \vec{\tau} \neq U_{\text {ext }} \cdot \vec{\tau} \text {. }
\end{aligned}
$$

Because of (3.13c), we may prefer not to correct the velocity when its divergence is small enough, instead of introducing errors at the boundary. This problem occurs in the resolution of every numerical methods that discretizes the equations of divergence-free fluids. However, one can show that this error reduces with $\Delta t$.

\section{NUMERICAL VALIDATION OF THE CONFINEMENT SCHEME}

The reflection scheme, detailed in Section 3 above, is aimed to confine the particles inside the domain $\mathcal{D}$, and to transfer information on Eulerian quantities available at an upper scale to the Lagrangian system in $\mathcal{D}$. The former is transmitted by means of a Dirichlet condition (1.2) on the velocity field. On a linear Lagrangian process, we first validate this downscaling method, and then study the impact of this scheme on the statistics of the process inside $\mathcal{D}$, more particularly the turbulent kinetic energy. 
Here, we consider a linear one dimensional Lagrangian model, namely an Ornstein-Uhlenbeck process for one component of the velocity field, and its primitive for the position. The corresponding free process $(Y, \mathcal{V})$ in $\mathbb{R} \times \mathbb{R}$ reads:

$$
\left\{\begin{array}{l}
Y_{t}=X_{0}+\int_{0}^{t} \mathcal{V}_{s} \mathrm{~d} s \\
\mathcal{V}_{t}=\mathcal{U}_{0}-c \int_{0}^{t}\left(\mathcal{V}_{s}-m\right) \mathrm{d} s+\sigma W_{t}
\end{array}\right.
$$

on some probability space $(\Omega, \mathcal{F}, \mathbb{P})$, equipped with a one-dimensional Brownian motion $W$. In the sequel, we refer to the process $(Y, \mathcal{V})$ as the Langevin-Ornstein-Uhlenbeck (LOU) process. The velocity component of this free process is spatially homogeneous. The velocity equation admits a Gaussian stationary solution. Moreover, the solution of equation (4.1) confined in $[0,+\infty)$, as well as its periodized version in the torus $\mathbb{T}=\mathbb{R} / L \mathbb{Z}$ for a fixed $L>0$, may be explicitly written, providing analytical expressions for the first and second conditional moments, that will allow us to study numerically the impact of the confinement terms in the first conditional moments.

For the sake of simplicity, we keep the same notation for the one dimensional confined Lagrangian process than for the SDM process, $(X, \mathcal{U})$.

In Section 4.1, we consider the case where the mean-revert parameter $m$ is zero and we use the analytical solution of equation (4.1) confined in $[0,+\infty)$ for a first numerical validation of both Dirichlet boundary conditions and variance behaviour, the latter corresponding to the turbulent kinetic energy in SDM. Using the one-dimensional version of the SDM scheme for confinement, we compare the asymptotic long-time behaviour of statistics with numerical simulations performed in a bounded interval $[0, L]$, where the confinement scheme is applied at both ends of the interval.

Next we investigate in Section 4.2 the case where the mean-revert $m$ is non-zero. This is of interest for the validation of SDM, since the mean-revert parameter $m$ is the analogous of the Eulerian velocity field $\langle U\rangle$ and must be compatible with the (non-zero) boundary conditions. Moreover the case $m \neq 0$ imposes to work with the pressure correction (corresponding to the optimal transport step correction in the SDM scheme) in the confined version of (4.1). We compare this case with the periodized version of $(4.1)$ in the interval $[0, L]$, the solution of this later case having its space-marginal uniformly distributed in $[0, L]$.

In all this section, we consider the initial variables $\left(X_{0}, \mathcal{U}_{0}\right)$ as a couple of independent random variables where $X_{0}$ is uniformly distributed in the interval $[0, L]\left(L>0\right.$ given), and $\mathcal{U}_{0}$ is a centered Gaussian variable with standard deviation $\sigma_{0}$. The parameters $L$ and $\sigma_{0}$ are respectively fixed to 10 and 0.1 in all the numerical simulations presented below.

\subsection{One dimensional SDM with zero Eulerian velocity field $(m=0)$}

As discussed in Remark 2.6, Section 2.2, the process $(X, \mathcal{U})$, confined in $[0,+\infty)$, solution of

$$
\left\{\begin{array}{l}
X_{t}=X_{0}+\int_{0}^{t} \mathcal{U}_{s} \mathrm{~d} s \\
\mathcal{U}_{t}=\mathcal{U}_{0}-c \int_{0}^{t} \mathcal{U}_{s} \mathrm{~d} s+\sigma W_{t}-\sum_{0<s \leq t} 2 \mathcal{U}_{s^{-}} \mathbb{1}_{\left\{X_{s}=0\right\}}
\end{array}\right.
$$

can be constructed from the free process $(Y, \mathcal{V})$ solution of

$$
\left\{\begin{array}{l}
Y_{t}=X_{0}+\int_{0}^{t} \mathcal{V}_{s} \mathrm{~d} s \\
\mathcal{V}_{t}=\mathcal{U}_{0}-c \int_{0}^{t} \mathcal{V}_{s} \mathrm{~d} s+\sigma W_{t}
\end{array}\right.
$$

Contrarily to SDM (see (1.1), (1.3) and (1.7) in Sect. 1), the model (4.2) does not carry any kind of pressure correction. As a consequence, the mass density (equivalent here to the space marginal of the probability density) associated with (4.2) has no more reason to be constant. 
We consider a rate of mean reversion $c>0$, and $\sigma>0$. As discussed in Section 2.2, the jump term in equation (4.2) models the confinement: the position $X_{t}$ is reflected with velocity $\mathcal{U}_{t}=-\mathcal{U}_{t^{-}}$when hitting the boundary $\{x=0\}$. It induces the Dirichlet condition $\mathbb{E}\left[\mathcal{U}_{t} / X_{t}=0\right]=0$, also called the mean no-permeability condition (see eq. (2.10)).

The probability density $\rho^{c}(t, x, u)$ of the confined particle $\left(X_{t}, \mathcal{U}_{t}\right)$ solution to $(4.2)$ is derived from the probability density $\rho(t, y, v)$ of the free process by identity $(2.21)$. The $n$-conditional moments on the velocity are then deduced from the free ones: for a.e. $(t, x) \in(0,+\infty) \times(0,+\infty)$,

$$
\mathbb{E}\left[\left(\mathcal{U}_{t}\right)^{n} / X_{t}=x\right]=\frac{\varrho(t, x) \mathbb{E}\left[\left(\mathcal{V}_{t}\right)^{n} / Y_{t}=x\right]+(-1)^{n} \varrho(t,-x) \mathbb{E}\left[\left(\mathcal{V}_{t}\right)^{n} / Y_{t}=-x\right]}{\varrho^{c}(t, x)},
$$

where $\varrho(t, x)$ and $\varrho^{c}(t, x)$ are respectively the mass density of the free process and the mass density of the confined process: $\varrho(t, x):=\int_{\mathbb{R}} \rho(t, x, v) \mathrm{d} v$ and $\varrho^{c}(t, x):=\int_{\mathbb{R}} \rho^{c}(t, x, v) \mathrm{d} v=\varrho(t, x)+\varrho(t,-x)$.

From identities (A.12) and (A.13) in Appendix A.2.1, we have the following analytical expressions for $\mathbb{E}\left[\mathcal{V}_{t} / Y_{t}=x\right]$ and $\mathbb{E}\left[\mathcal{V}_{t}^{2} / Y_{t}=x\right]:$ for all $t>0$,

$$
\begin{aligned}
\mathbb{E}\left[\mathcal{V}_{t} / Y_{t}=x\right]= & \frac{\mu(t)}{\nu_{1}(t)} x+\frac{\gamma(t) \sigma_{0}^{2} \exp (-c t)+\mu(t)}{\nu_{1}(t)+\left(\gamma(t) \sigma_{0}\right)^{2}} \frac{\mathbb{E}\left[\left(x-X_{0}\right) g_{\sigma_{1}(t)}\left(x-X_{0}\right)\right]}{\varrho(t, x)}, \\
\mathbb{E}\left[\mathcal{V}_{t}^{2} / Y_{t}=x\right]= & \frac{|\Sigma|(t)}{\nu_{1}(t)}+\frac{\nu_{1}(t)\left(\gamma(t) \sigma_{0}\right)^{2}}{\nu_{1}(t)+\left(\gamma(t) \sigma_{0}\right)^{2}}\left(\frac{\exp (-c t)}{\gamma(t)}-\frac{\mu(t)}{\nu_{1}(t)}\right)^{2} \\
& +\left(\frac{\gamma(t) \sigma_{0}^{2} \exp (-c t)+\mu(t)}{\nu_{1}(t)+\left(\gamma(t) \sigma_{0}\right)^{2}}\right)^{2} \frac{\mathbb{E}\left[\left(x-X_{0}\right)^{2} g_{\sigma_{1}(t)}\left(x-X_{0}\right)\right]}{\varrho(t, x)},
\end{aligned}
$$

where $g_{\sigma}$ denotes the one dimensional Gaussian function with standard deviation $\sigma$, the functions $\gamma(t), \Sigma(t)$, $\nu(t), \mu(t)$ are defined in the beginning of Appendix A.1 in (A.2), and $\sigma_{1}(t)$ in the beginning of Section A.2.

A straightforward calculation leads to

$$
\begin{aligned}
\mathbb{E}\left[\mathcal{U}_{t} / X_{t}=x\right]= & \frac{\mu(t)}{\nu_{1}(t)} x+\frac{\gamma(t) \sigma_{0}^{2} \exp (-c t)+\mu(t)}{\nu_{1}(t)+\left(\gamma(t) \sigma_{0}\right)^{2}} x+\frac{\gamma(t) \sigma_{0}^{2} \exp (-c t)+\mu(t)}{\nu_{1}(t)+\left(\gamma(t) \sigma_{0}\right)^{2}} \\
& \times \frac{\mathbb{E}\left[X_{0}\left(g_{\sigma_{1}(t)}\left(x-X_{0}\right)-g_{\sigma_{1}(t)}\left(x+X_{0}\right)\right)\right]}{\varrho^{c}(t, x)},
\end{aligned}
$$

and

$$
\begin{aligned}
\mathbb{E}\left[\mathcal{U}_{t}^{2} / X_{t}=x\right]= & \frac{|\Sigma|(t)}{\nu_{1}(t)}+\frac{\nu_{1}(t)\left(\gamma(t) \sigma_{0}\right)^{2}}{\nu_{1}(t)+\left(\gamma(t) \sigma_{0}\right)^{2}}\left(\frac{\exp (-c t)}{\gamma(t)}-\frac{\mu(t)}{\nu_{1}(t)}\right)^{2} \\
& +\left(\frac{\gamma(t) \sigma_{0}^{2} \exp (-c t)+\mu(t)}{\nu_{1}(t)+\left(\gamma(t) \sigma_{0}\right)^{2}}\right)^{2} \frac{\mathbb{E}\left[\left(x-X_{0}\right)^{2} g_{\sigma_{1}(t)}\left(x-X_{0}\right)\right]}{\varrho^{c}(t, x)}
\end{aligned}
$$

from which we can easily compute the long-time statistics $\mathbb{E}\left[\mathcal{U}_{t} / X_{t}=x\right]$ and $\mathbb{E}\left[\mathcal{U}_{t}^{2} / X_{t}=x\right]$, as long as we consider a bounded conditional position $0 \leq x \leq L$ and the initial variable $\left|X_{0}\right|$ bounded $\mathbb{P}$ almost everywhere. We identify two constants $C_{1}$ and $C_{2}$, depending on $x$ such that

$$
\begin{aligned}
\langle U\rangle(t, x) & =\frac{C_{1}}{2 t}+o\left(\frac{1}{t}\right), \\
\left\langle u^{2}\right\rangle(t, x)-\frac{\sigma^{2}}{2 c} & =\frac{C_{2}}{t}+o\left(\frac{1}{t}\right) .
\end{aligned}
$$


For the first and second conditional moments $\mathbb{E}\left[\mathcal{U}_{t} / X_{t}=x\right]$ and $\mathbb{E}\left[\mathcal{U}_{t}^{2} / X_{t}=x\right]$, the long-time behaviour of the confined process $\left(X_{t}, \mathcal{U}_{t}\right)$ is equivalent with the free process $\left(Y_{t}, \mathcal{V}_{t}\right)$ (see Rem. A.2 in the Appendix). The limit values are the mean and variance of the equilibrium Gaussian law $\mathcal{N}\left(0, \frac{\sigma^{2}}{2 c}\right)$ of the free velocity $\mathcal{V}_{t}$.

\subsubsection{First numerical reference and validation}

The numerical computation of conditional moments of the confined model (4.2) is difficult to achieve because the support of the law of the particles is the unbounded half-line. When $L$ is sufficiently large, the two end confinement points of $[0, L]$ behave (almost) independently, as a superposition of two half-line confinements. Hence, the following process confined in $[0, L]$

$$
\left\{\begin{array}{l}
X_{t}=X_{0}+\int_{0}^{t} \mathcal{U}_{s} \mathrm{~d} s \\
\mathcal{U}_{t}=\mathcal{U}_{0}-c \int_{0}^{t} \mathcal{U}_{s} \mathrm{~d} s+\sigma W_{t}-\sum_{0<s \leq t} 2 \mathcal{U}_{s^{-}}\left(\mathbb{1}_{\left\{X_{s}=0\right\}}+\mathbb{1}_{\left\{X_{s}=L\right\}}\right)
\end{array}\right.
$$

appears to be the nearest model numerically solvable that can reproduce the asymptotic behaviours (4.3) and (4.4).

We simulated particles with an adapted one dimensional version of SDM's algorithm (see Sect. 3.2). We use an explicit exponential Euler scheme (see (3.7)), and apply the confinement procedure (3.8), (3.9) with $U_{\text {ext }}=0$. We smooth the confinement effect with the regularization function $h_{\delta}$ as described in (3.3).

Comparisons are made on the first moment $\langle U\rangle$ and its variance $\left\langle u^{2}\right\rangle$ and their asymptotic long-time analytical expressions, equivalent to (4.3) and (4.4). The final time simulation $T$ is taken equal to $T=100$. At time $t=0$, $N_{p}=N_{c} N_{p c}$ particles are uniformly distributed in the interval $[0, L]$, with velocity randomly generated with a Gaussian law $\mathcal{N}\left(0, \sigma_{0}\right)$. The initial variance $\sigma_{0}^{2}=0.01$ is chosen smaller than the expected asymptotic variance $\sigma^{2} / 2 c$, where $\sigma$ and $c$ are $O(1)$.

In all the figures below, mean values are shown in each cell center. Except in Figure 4 where several $N_{c}$ and $\delta$ are compared, we fix $N_{c}=200$ and $\delta=L / N_{c}=0.05$. The smoothing parameter $\delta$ of the confinement corresponds then to the size of the boundary cell. The time step $\Delta t=0.01$ is chosen sufficiently small so that particles do not cross more than one cell during a time step. The number of particles per cell is fixed to $N_{p c}=10000$, in order the Monte Carlo error (3.6) to be small enough.

As we can observe in Figure 2 with $U_{\text {ext }}(0)=U_{\text {ext }}(L)=0$, the numerical first and second conditional moments provided by our scheme match the analytical results given in (4.3) and (4.4). The particle reflection at the boundaries affects neither the first moment nor the variance, and these values are in agreement with the theoretical prediction: the variance $\left\langle u^{2}\right\rangle(T, x)$ is almost constant in $[0, L]$ and matches the value $\sigma^{2} / 2 c$. The Monte Carlo noise on $\langle U\rangle$ remains in the asymptotic confident interval of $\left\langle u^{2}\right\rangle$

$$
\mathbb{P}\left(\langle U\rangle-\langle U\rangle_{\mathrm{SDM}} \in\left[-\frac{\sqrt{2} \sigma}{\sqrt{c N_{p c}}}, \frac{\sqrt{2} \sigma}{\sqrt{c N_{p c}}}\right]\right) \geq 95 \% .
$$

\subsubsection{Effect of non-homogeneous boundary conditions}

In order to test the behaviour of the confinement scheme on an irregular case, we have simulated the confined Langevin system when the boundary conditions are no more compatible with the mean revert parameter $m$. The corresponding equation should be typically,

$$
\left\{\begin{array}{l}
X_{t}=X_{0}+\int_{0}^{t} \mathcal{U}_{s} \mathrm{~d} s \\
\mathcal{U}_{t}=\mathcal{U}_{0}-c \int_{0}^{t} \mathcal{U}_{s} \mathrm{~d} s+\sigma W_{t}+\sum_{0<s \leq t} 2\left(U_{\text {ext }}\left(X_{s}\right)-\mathcal{U}_{s^{-}}\right)\left(\mathbb{1}_{\left\{X_{s}=0\right\}}+\mathbb{1}_{\left\{X_{s}=L\right\}}\right)
\end{array}\right.
$$



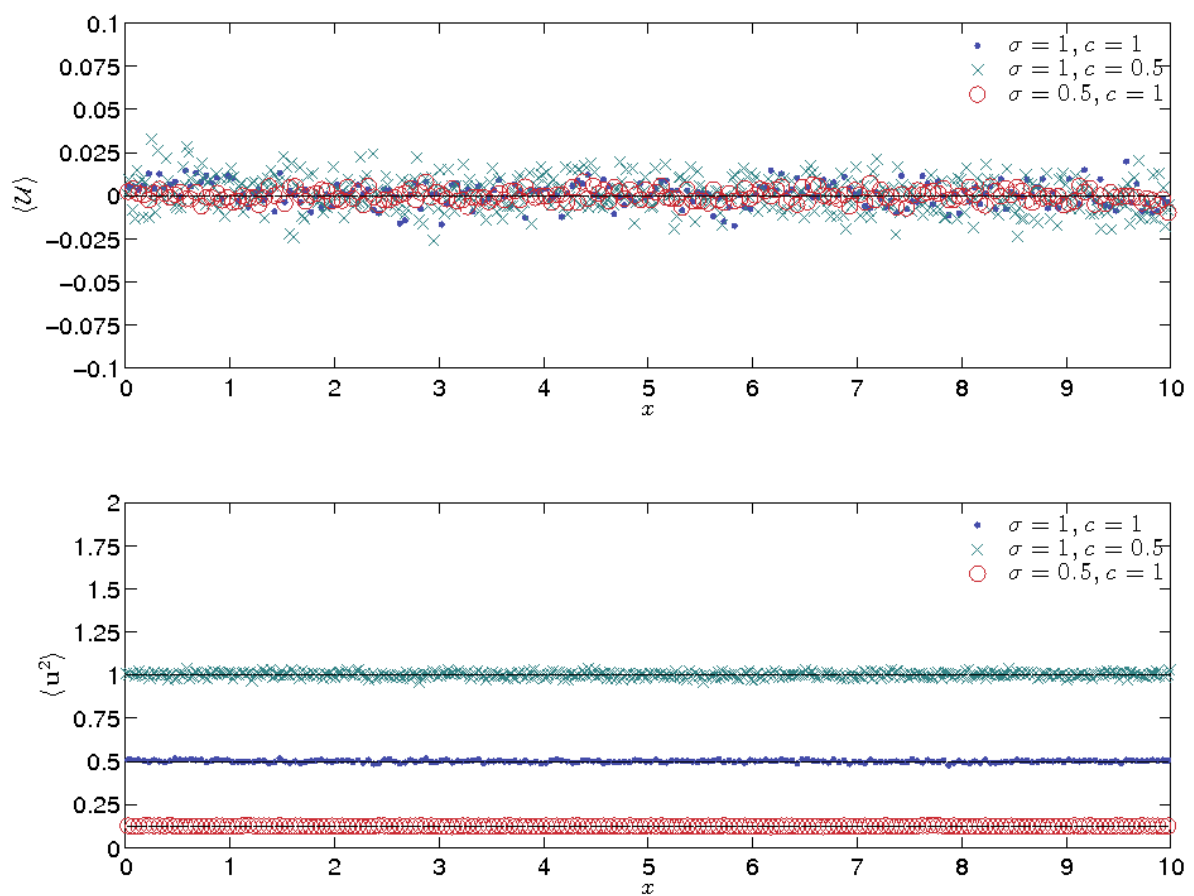

Figure 2. The homogeneous case $m=U_{\text {ext }}(0)=U_{\text {ext }}(L)=0$. The $\langle U\rangle(T, \cdot)$ and $\left\langle u^{2}\right\rangle(T, \cdot)$ behaviour in the confined interval $[0, L]$ from the simulation of system (4.5). The theoretical asymptotic values are plotted in continuous lines.

with non zero $U_{\text {ext }}(0), U_{\text {ext }}(L)$. Even if the well-posedness of (4.6) is unclear, such an experiment gives an idea of the variance perturbation due to the forcing. We let unchanged the set of numerical parameters $\left\{T, \Delta t, N_{c}, N_{p c}, \delta\right\}$. As Figure 3 evidences for two given sets of non-zero boundary conditions, the numerical first moment fails to return with precision the boundary forcing, although this can be modulated with the choice of $N_{p c}$ and $\delta$. Even for $T=100$, the size of the computational domain $L$ is clearly large enough to separate the left and right forcing effects. The perturbation of the variance depends strongly on the rarefaction effect or the concentration effect the forcing locally produces on the particle distribution.

In the next section, we pursue our analysis, by introducing the pressure correction term (and hence the constant mass density constraint) in the confining equation.

\subsection{One dimensional SDM with non-zero Eulerian velocity field $(\boldsymbol{m} \neq \mathbf{0})$}

Consider now the LOU type process (4.1) with a non zero mean revert parameter $m$. In that case, as pointed out in Remark A.1, the Eulerian velocity of the free process behaves like $\mathbb{E}\left[\mathcal{V}_{t} / Y_{t}=y\right] \simeq m / 2$ as $t \rightarrow+\infty$, that prevents from interpreting equation (4.1) as the linearized version of

$$
\left\{\begin{array}{l}
Y_{t}=X_{0}+\int_{0}^{t} \mathcal{V}_{s} \mathrm{~d} s \\
\mathcal{V}_{t}=\mathcal{U}_{0}-c \int_{0}^{t}\left(\mathcal{V}_{s}-\mathbb{E}\left[\mathcal{V}_{s} / Y_{s}\right]\right) \mathrm{d} s+\sigma W_{t}
\end{array}\right.
$$

where we want to replace $\mathbb{E}\left[\mathcal{V}_{t} / Y_{t}=y\right]$ by $m$ as a numerical test case. Moreover, the simulation of confined version of (4.1) is difficult to achieve as the forcing and $m \neq 0$ may produce a concentration of particles at a boundary. For those reasons, we modify equation (4.1) with the pressure correction term. A mathematical 

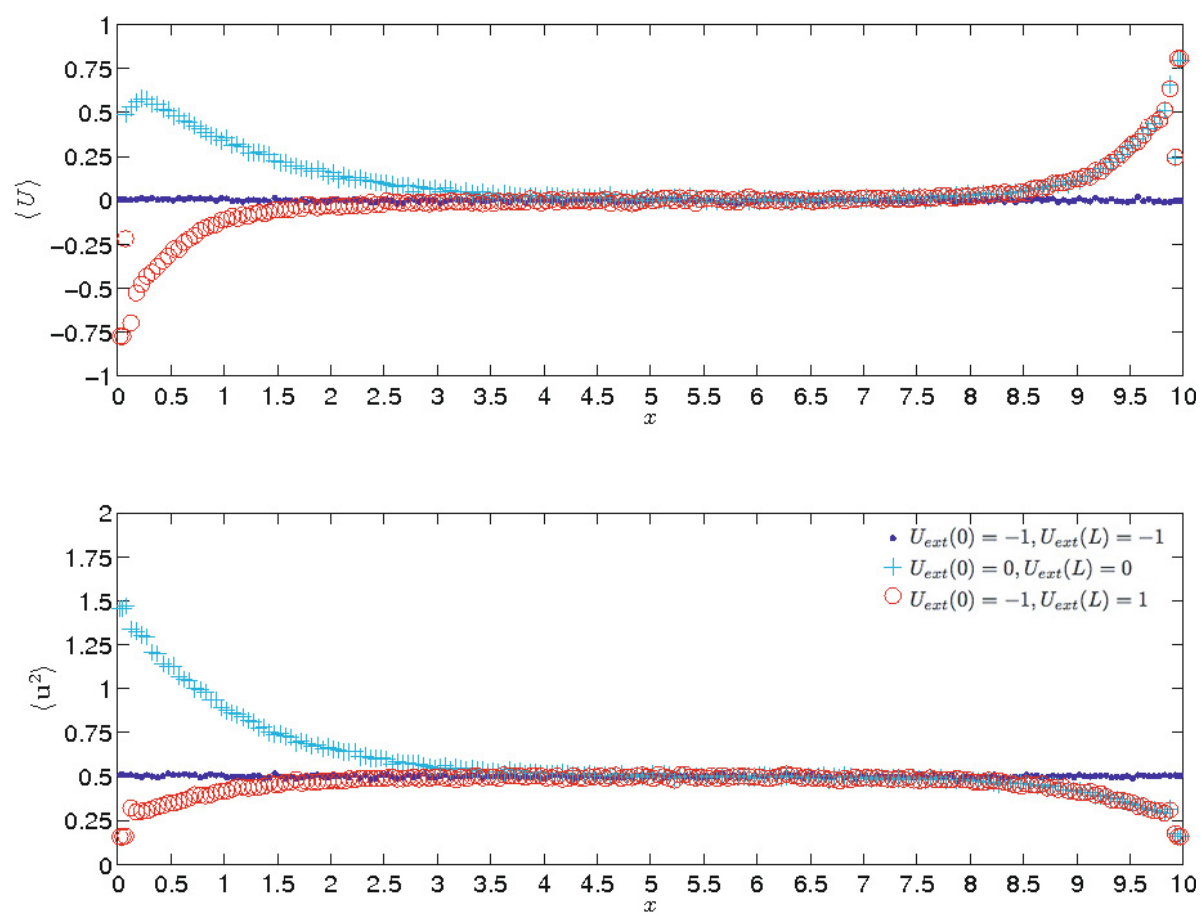

Figure 3. Inhomogeneous Dirichlet conditions and $m=0$. The $\langle U\rangle(T, \cdot)$ and $\left\langle u^{2}\right\rangle(T, \cdot)$ behaviour in the confined interval $[0, L]$ from the simulation of system (4.6). Three sets of boundary forcing are applied at $x=0$ and $x=L$.

analysis of the Poisson equation's role coupled with (4.7) is proposed in [3]. The authors study equations of type

with

$$
\left\{\begin{array}{l}
Y_{t}^{\mathbb{T}}=\left[X_{0}+\int_{0}^{t} \mathcal{V}_{s} \mathrm{~d} s\right] \bmod L \\
\mathcal{V}_{t}=\mathcal{U}_{0}+\int_{0}^{t}\left(\mathbb{E}\left[\mathcal{V}_{s} / Y_{s}^{\mathbb{T}}\right]-\mathcal{V}_{s}-\nabla P\left(s, Y_{s}^{\mathbb{T}}\right)\right) \mathrm{d} s+\sigma W_{t},
\end{array}\right.
$$

$$
-\triangle_{x} P(t, x)=\sum_{i, j=1}^{d} \partial_{i j}^{2}\left(\mathbb{E}\left(\mathcal{V}_{t}^{(i)} \mathcal{V}_{t}^{(j)} / Y_{t}^{\mathbb{T}}=x\right)\right), \quad(t, x) \in(0,+\infty) \times \mathbb{T} .
$$

Here, $\mathbb{T}$ is the torus $\mathbb{R} / L \mathbb{Z}$ and $[x] \bmod L:=x-L\lfloor x / L\rfloor$ where $\lfloor x\rfloor$ is the integer part of $x \in \mathbb{R}$. In particular, it is shown that $Y_{t}^{\mathbb{T}}$ stays uniformly distributed in $[0, L]$ as soon as $X_{0}$ is.

As show in Appendix B, the periodic-LOU process

$$
\left\{\begin{array}{l}
Y_{t}^{\mathbb{T}}=\left[X_{0}+\int_{0}^{t} \mathcal{V}_{s} \mathrm{~d} s\right] \bmod L \\
\mathcal{V}_{t}=\mathcal{U}_{0}-c \int_{0}^{t}\left(\mathcal{V}_{s}-m\right) \mathrm{d} s+\sigma W_{t}
\end{array}\right.
$$

is also stationary in its space variable: $Y_{t}^{\mathbb{T}}$ is uniformly distributed in $[0, L]$ for all $t \geq 0$. Moreover $\mathbb{E}\left[\mathcal{V}_{t} / Y_{t}^{\mathbb{T}}=y\right]$ tends to $m$ exponentially fast, and

$$
\mathbb{E}\left[\mathcal{V}_{t}^{2} / Y_{t}^{\mathbb{T}}=y\right] \rightarrow m^{2}+\frac{\sigma^{2}}{2 c} \text { when } t \rightarrow+\infty
$$



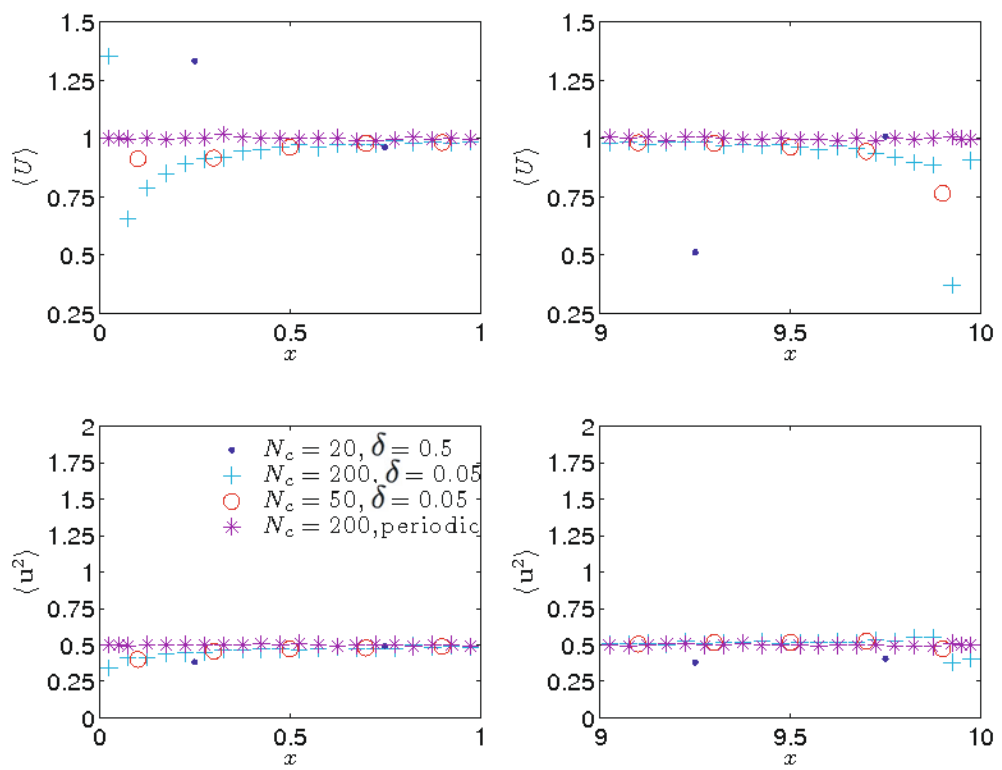

Figure 4. For $m=U_{\text {ext }}(0)=U_{\text {ext }}(L)=1,\langle U\rangle$ and $\left\langle u^{2}\right\rangle$ for various number of cells, with a constant number of particles per cell, and $\sigma=c=1$.

\subsubsection{Numerical validation for a non-zero mean-revert $m \neq 0$}

In this section, we study the effect of the forcing confinement combined with the pressure correction. For this purpose, we have simulated the confined system

$$
\left\{\begin{array}{l}
X_{t}=X_{0}+\int_{0}^{t} \mathcal{U}_{s} \mathrm{~d} s \\
\mathcal{U}_{t}=\mathcal{U}_{0}+\int_{0}^{t}\left[c\left(m-\mathcal{U}_{s}\right)-\nabla P\left(s, X_{s}\right)\right] \mathrm{d} s+\sigma W_{t}+\sum_{0<s \leq t} 2\left(U_{\text {ext }}\left(X_{s}\right)-\mathcal{U}_{s^{-}}\right)\left(\mathbb{1}_{\left\{X_{s}=0\right\}}+\mathbb{1}_{\left\{X_{s}=L\right\}}\right)
\end{array}\right.
$$

coupled with

$$
-\triangle_{x} P(t, x)=\sum_{i, j=1}^{d} \partial_{i j}^{2}\left(\mathbb{E}\left(\mathcal{U}_{t}^{(i)} \mathcal{U}_{t}^{(j)} / X_{t}=x\right)\right), \quad(t, x) \in(0,+\infty) \times[0, L] .
$$

We compare it with the simulations of (4.8) in order to try to discriminate between the effects of the boundary conditions and the effect of the constant mass density constraint.

As previously, we simulated particles using an adapted version of SDM's algorithm for the one dimensional case. It has to be underlined here that at each time step, the constant mass density constraint (see Step 3 of the numerical scheme in Sect. 3.2), is applied to overcome the Poisson equation. In one dimension, the optimal transport scheme resulting from this constraint is solved exactly, using a sorting procedure [31].

In the numerical simulations, the size of each cell is equal to $\delta: N_{c} \delta=L$, each cell containing $N_{p c}=10000$ particles.

Results are shown in Figure 4, near the boundaries, for $x \in[0,1]$ (left), and [9, 10] (right), for several values of $\delta$. In all the interval $[0, L]$, the variance behaves accordingly to equation (4.4). Zooming at the two boundaries reveals a singular behaviour of the mean velocity, depending on the sign of $U_{\text {ext }}$.

Here, we point out that, with our numerical scheme, variances (or equivalently the turbulent kinetic energy) are barely influenced by the boundaries. This tends to show that our numerical algorithm is well adapted 
to a downscaling method (with boundary forcing): the confinement leads to a rather little perturbation of the turbulent kinetic energy $k$, which is a central physical variable.

For the first velocity moment, a rather sharp variation of $\langle U\rangle$ appears near the boundary (see Fig. 4). For instance, in top right figure, the first blue cross value on the right returns rather goodly the Dirichlet condition $\langle U\rangle=1$, the blue cross values inside the interval are coherent with the value $m=1$. But the second blue cross value on the right reveals the jump for reflected particles: one can assimilate the peaks observed numerically as a stronger impact of the characteristic function $h_{\delta}$ on $\langle U\rangle$. Indeed, in the top right figure, the outgoing flow (since $m=U_{\text {ext }}=1$ ) accumulates the particles at the boundary $x=10$ in the delta-zone, and the optimal transport pushes the particle inside, with boundary information that are less meaningful inside the domain. At the opposite boundary $x=0$ (top left figure), the entering flow brings particles naturally out the delta-zone.

Next, we deduced from a fine simulation $\left(N_{c}=200\right)$ a coarser one $\left(N_{c}=50\right)$, for the same size of confining zone $\delta=0.05$. This coarse simulation, plotted with red circles in Figure 4, aims to show the smoothing effect when the $\delta$-zone of the confining numerical scheme is chosen smaller than the cell size at the boundary. The computation of the Eulerian velocity at the boundary involves all the particles in the boundary cell, and not only the ones in the $\delta$-zone. We compute the coarse estimation in the following way: given the simulated particles at time $T=100$ for $N_{c}=200$ (corresponding to a cell's size of 0.05), we consider a four times bigger cell discretization of $[0, L]$ : for $j=1$ to 50 , at the center of coarse cell $\bar{x}_{j}=L(j-0.5) / 50$, we compute:

$$
\langle U\rangle^{N_{c}=50}\left(\bar{x}_{j}\right)=\frac{4}{N_{c}} \sum_{k=1}^{N_{p}}\langle U\rangle^{N_{c}=200}\left(X_{T}^{k}\right)_{\left\{X_{T}^{k} \in \mathcal{C}_{j}\right\}} .
$$

The coarser simulation then leads to a rather regular mean velocity $\langle U\rangle$. Obviously, when the peak is sharp, as in top right figure, the mean between Dirichlet condition and mean velocity inside the interval remains important too.

In the next paragraph, another set of simulations goes into further details on the impact of the optimal transport procedure at the boundary.

\subsubsection{Impact of the forcing $U_{\mathrm{ext}}$ on $\langle U\rangle$ and $\left\langle u^{2}\right\rangle$ at the boundary}

Let us now investigate the impact of the boundary conditions $U_{\text {ext }}$ on the first and second moments of the velocity at the boundary of the domain. In the simple case of a one-way coupling in which the information only goes from the large scales to the small ones, the forcing terms $U_{\text {ext }}$ really impose their value to $\langle U\rangle$ computed at the boundary. In this case, the numerical values of $U_{\text {ext }}$ and $m$ should thus be compatible. However, when one considers a two-way coupling in which the local model is not only a refinement, but also a correction of the large scale information, then there might be (or should be) a difference between the external (coarse) velocity $U_{\text {ext }}$ and the computed values of $\langle U\rangle$ at the boundary. This suggests the study (at least numerically) of (4.9) when $U_{\text {ext }}$ and $m$ have different values.

Figure 5 shows the final value (at $T=100$ ) at the right boundary of the mean velocity and its variance when $U_{\text {ext }}(L)$ takes values from -3 to 3 , with different values for the parameter $m$. The values of $\sigma$ and $c$ are unchanged, so that the expected variance is $\sigma^{2} / 2 c=0.5$. One can see that the second moment is well approximated by the numerical scheme when $U_{\text {ext }}(L)>0$ (outgoing velocity). This can be explained by the fact that a sufficient number of particles are carried in the boundary neighborhood for the Monte-Carlo approximation to converge. When $U_{\text {ext }}(L)<0$ (incoming velocity), the particles are chased from the boundary to the interior of the domain and the second moments are poorly approximated (except when $m<0$ ).

More generally, looking both at the first and second moments, we notice that the numerical scheme behaves pretty well when $U_{\text {ext }}(L)=m$, which was expected, but that the results are also satisfactory when $U_{\text {ext }}(L)$ and $m$ have compatible (though different) values: for example $U_{\text {ext }}(L)=2$ and $m=1$. However, mean value and/or variance at the boundary is not correct for incompatible values of $U_{\text {ext }}(L)$ and $m$ : see the cases $(-3,1)$ or $(3,-1)$. 

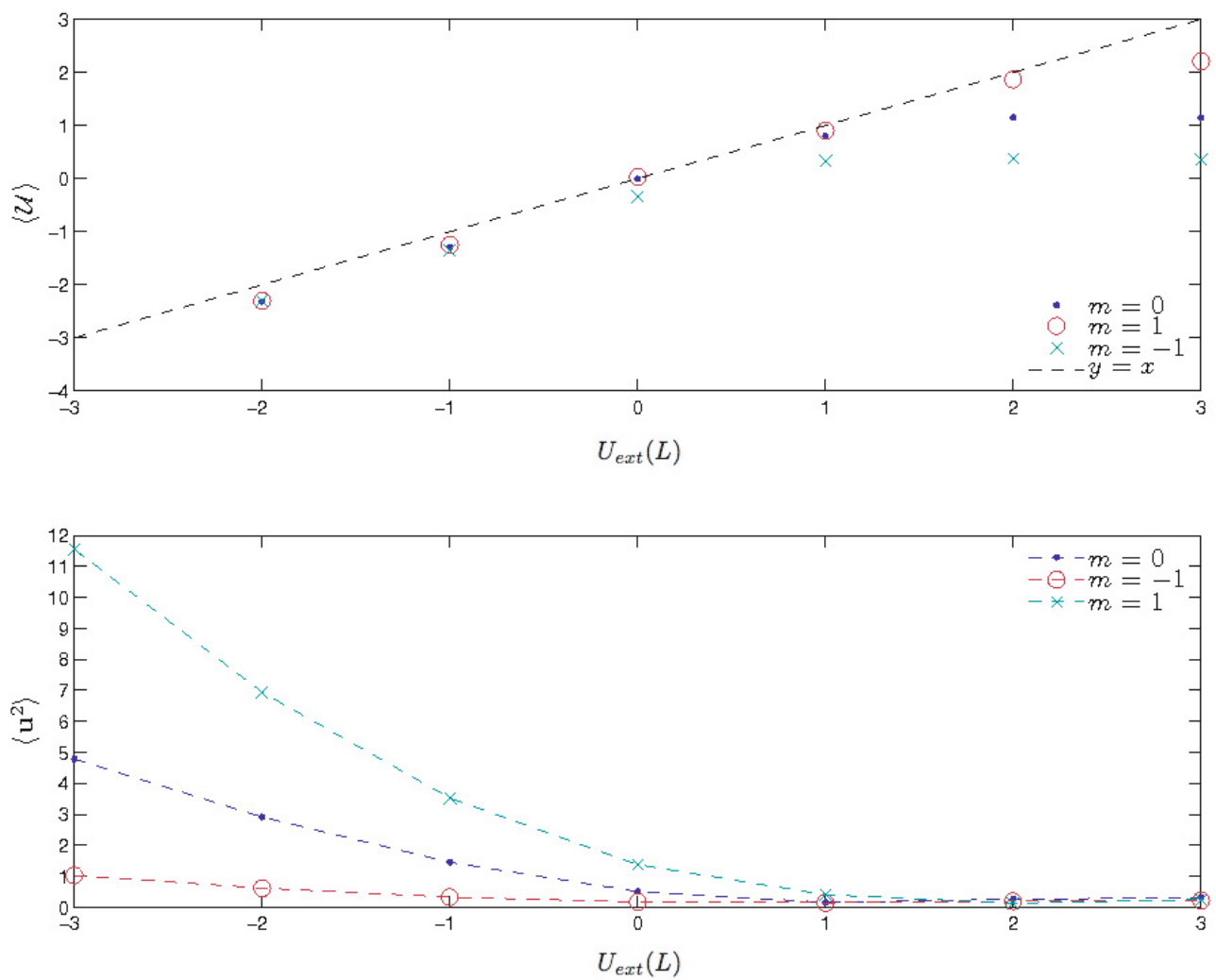

Figure 5 . For $m=-1,0$ or $m=1$, behaviour of the boundary value of $\langle U\rangle$ and $\left\langle u^{2}\right\rangle$ respectively to the guidance $U_{\text {ext }}$ at $x=L$.

\subsection{Conclusion}

Let us now conclude with some numerical results for the complete 3D-SDM model. The simulations presented in Figure 6 correspond to equations (1.1), (1.3), (1.7), discretized thanks to the numerical scheme introduced in Section 3. The external forcing terms used for the computations of Figure 6 correspond to the (idealized) case where the external field $U_{\text {ext }}$ depends neither on $t>0$, nor on $x \in \partial \mathcal{D}$. As a consequence, the equation for the production of turbulent kinetic energy

$$
\frac{\partial k}{\partial t}=\text { thermal production }+ \text { transport }+ \text { shear }- \text { dissipation } \varepsilon
$$

reduces (thanks to eq. (1.3)) to

$$
\frac{\partial k}{\partial t}=-\frac{C_{\varepsilon}}{\ell_{m}} k^{3 / 2}
$$

The numerical behaviour for the turbulent kinetic energy in Figure 6 is thus the one expected from equation (4.10). We also notice that, as it was diagnosed for the $1 \mathrm{D}$ case (see Sect. 4), the numerical scheme does take the boundary conditions into account, as evidenced in Figure 6.

Naturally, these first very encouraging results only insure a partial validation of our model: they will have to be combined with numerical simulations in more realistic cases (e.g. with vertical shearing). Our model will also have to be improved, in particular for the stratification effects. This will be presented in further studies. 


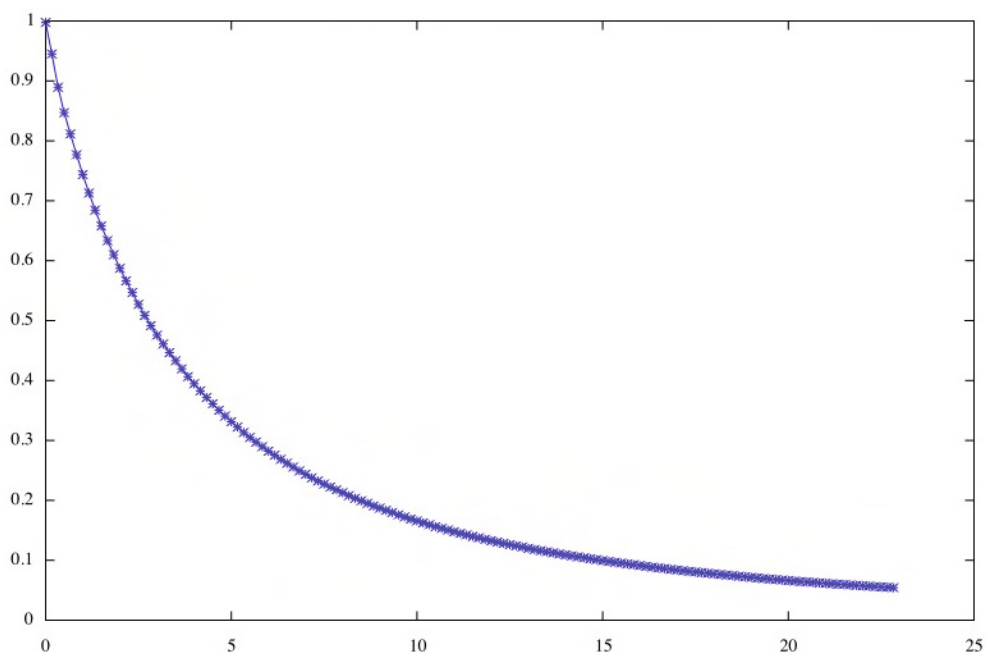

(a)

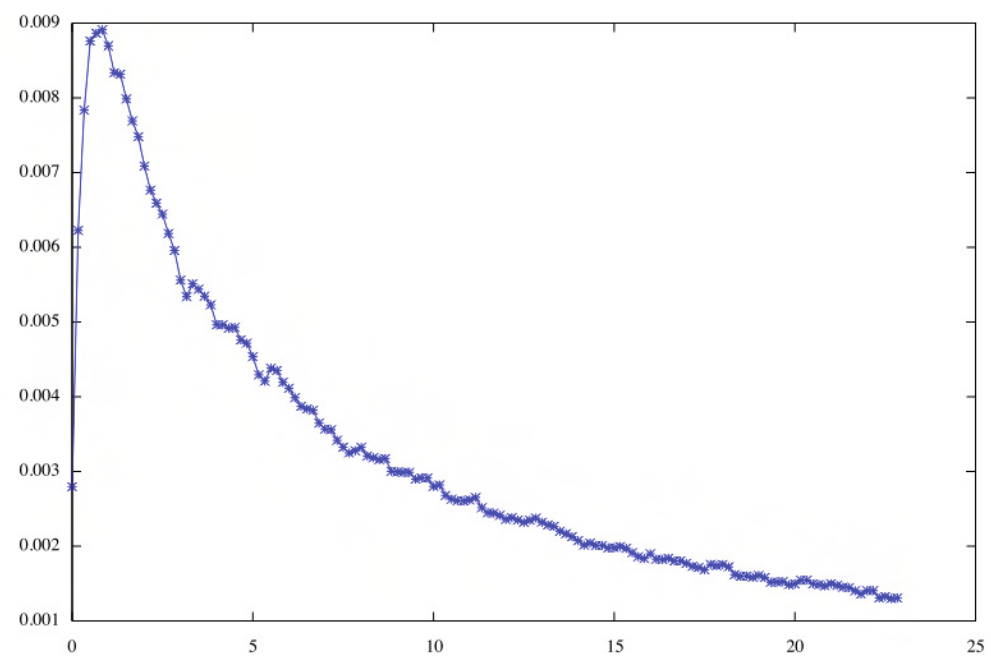

(b)

FiguRE 6. Idealized simulation of SDM with constant boundary forcing terms. (a) Time evolution of the space-averaged turbulent kinetic energy $\|k\|_{L^{1}(\mathcal{D})}$. (b) Time evolution of the $L^{2}$ norm of the zonal velocity boundary error $\left\|U_{\text {ext }}-\langle U\rangle_{S D M}\right\|_{L^{2}(\partial \mathcal{D})}$.

\section{A. The Free Langevin-Ornstein-Uhlenbeck PRocess}

The free Langevin-Ornstein-Uhlenbeck process $(Y, \mathcal{V})$ on $\mathbb{R} \times \mathbb{R}$ is the solution to the following system of stochastic differential equations

$$
\left\{\begin{array}{l}
Y_{t}=Y_{0}+\int_{0}^{t} \mathcal{V}_{s} \mathrm{~d} s \\
\mathcal{V}_{t}=V_{0}-c \int_{0}^{t}\left(\mathcal{V}_{s}-m\right) \mathrm{d} s+\sigma W_{t} .
\end{array}\right.
$$


On a probability space $(\Omega, \mathcal{F}, \mathbb{P})$, given a one dimensional Brownian motion $W$ and initial random variables $\left(Y_{0}, V_{0}\right)$ for the position and velocity, the solution of system (A.1) writes

$$
\begin{aligned}
& Y_{t}=Y_{0}+\frac{V_{0}-m}{c}(1-\exp (-c t))+m t+\frac{\sigma}{c} \int_{0}^{t}(1-\exp (-c(t-s))) \mathrm{d} W_{s}, \\
& \mathcal{V}_{t}=m+\left(V_{0}-m\right) \exp (-c t)+\sigma \int_{0}^{t} \exp (-c(t-s)) \mathrm{d} W_{s} .
\end{aligned}
$$

In this appendix section, we derive explicit expressions for the conditional moments $\mathbb{E}\left[\mathcal{V}_{t} / Y_{t}=y\right]$ and $\mathbb{E}\left[\mathcal{V}_{t}^{2} / Y_{t}=y\right]$, for $t>0$. We assume that the initial condition $\left(Y_{0}, V_{0}\right)$ is a couple of independent random variables having finite second moments.

\section{A.1. General initial conditions}

As a preliminary computation, we consider the following degenerate case where $\left(Y_{0}, V_{0}\right)$ is deterministic, equal to $\left(y_{0}, v_{0}\right)$. Denoting by $\left(\bar{Y}\left(t, y_{0}, v_{0}\right), \bar{V}\left(t, y_{0}, v_{0}\right)\right)$ the means $\left(\mathbb{E}_{\left(y_{0}, v_{0}\right)}\left[Y_{t}\right], \mathbb{E}_{\left(y_{0}, v_{0}\right)}\left[\mathcal{V}_{t}\right]\right)$, we have

$$
\begin{aligned}
& \bar{Y}\left(t, y_{0}, v_{0}\right)=y_{0}+v_{0} \gamma(t)+m(t-\gamma(t)), \\
& \bar{V}\left(t, v_{0}\right)=v_{0} \exp (-c t)+m c \gamma(t), \quad \text { for } \gamma(t):=\frac{1}{c}(1-\exp (-c t)),
\end{aligned}
$$

and denoting by $\Sigma(t)$ the covariance matrix of this Gaussian vector $\left(Y_{t}, \mathcal{V}_{t}\right)$, which is positive definite and invertible for all $t>0$,

$$
\Sigma(t)=\left(\begin{array}{cc}
\nu_{1}(t) & \mu(t) \\
\mu(t) & \nu_{2}(t)
\end{array}\right)=\left(\begin{array}{cc}
\frac{\sigma^{2}}{c^{2}} t+\frac{\sigma^{2}}{2 c^{3}}(4(\exp (-c t)-1)+1-\exp (-2 c t)) & \frac{\sigma^{2}}{2 c^{2}}(1-\exp (-c t))^{2} \\
\frac{\sigma^{2}}{2 c^{2}}(1-\exp (-c t))^{2} & \frac{\sigma^{2}}{2 c}(1-\exp (-2 c t))
\end{array}\right)
$$

the joint probability density $\rho$ for the vector $\left(Y_{t}, \mathcal{V}_{t}\right)$ at time $t$ starting from $\left(y_{0}, v_{0}\right)$ at times 0 , writes

$$
\begin{aligned}
\rho\left(0, y_{0}, v_{0} ; t, y, v\right)= & \frac{1}{2 \pi|\Sigma(t)|^{1 / 2}} \exp \left(-\frac{1}{2|\Sigma(t)|}\left(\nu_{2}(t)\left(y-\bar{Y}\left(t, y_{0}, v_{0}\right)\right)^{2}-2 \mu(t)\left(y-\bar{Y}\left(t, y_{0}, v_{0}\right)\right)\right.\right. \\
& \left.\left.\times\left(v-\bar{V}\left(t, v_{0}\right)\right)+\nu_{1}(t)\left(v-\bar{V}\left(t, v_{0}\right)\right)^{2}\right)\right) .
\end{aligned}
$$

Its space-marginals $\varrho\left(0, y_{0}, v_{0} ; t, x\right)=\int_{\mathbb{R}} \rho\left(0, y_{0}, v_{0} ; t, y, v\right) \mathrm{d} v$ writes, for all $t>0$,

$$
\varrho\left(0, y_{0}, v_{0} ; t, y\right)=\frac{1}{\sqrt{2 \pi \nu_{1}(t)}} \exp \left(-\frac{1}{2 \nu_{1}(t)}\left(y-\bar{Y}\left(t, y_{0}, v_{0}\right)\right)^{2}\right)
$$

so that

$\rho\left(0, y_{0}, v_{0} ; t, y, v\right)=\varrho\left(0, y_{0}, v_{0} ; t, y\right) \frac{\sqrt{\nu_{1}(t)}}{\sqrt{2 \pi|\Sigma(t)|}} \exp \left(-\frac{\nu_{1}(t)}{2|\Sigma(t)|}\left(\left(v-\bar{V}\left(t, v_{0}\right)\right)-\frac{\mu(t)}{\nu_{1}(t)}\left(y-\bar{Y}\left(t, y_{0}, v_{0}\right)\right)\right)^{2}\right)$. 
From this last expression, we immediately get the conditional moments $\mathbb{E}_{\left(y_{0}, v_{0}\right)}\left[\mathcal{V}_{t} / Y_{t}=y\right]$ and $\mathbb{E}_{\left(y_{0}, v_{0}\right)}\left[\mathcal{V}_{t}^{2} / Y_{t}=y\right]$ : for all $t>0$,

$$
\begin{aligned}
& \mathbb{E}_{\left(y_{0}, v_{0}\right)}\left[\mathcal{V}_{t} / Y_{t}=y\right]=\frac{\int_{\mathbb{R}} v \rho\left(0, y_{0}, v_{0} ; t, y, v\right) \mathrm{d} v}{\varrho\left(0, y_{0}, v_{0} ; t, y\right)}=\bar{V}\left(t, v_{0}\right)+\frac{\mu(t)}{\nu_{1}(t)}\left(y-\bar{Y}\left(t, y_{0}, v_{0}\right)\right), \\
& \mathbb{E}_{\left(y_{0}, v_{0}\right)}\left[\mathcal{V}_{t}^{2} / Y_{t}=y\right]=\frac{\int_{\mathbb{R}} v^{2} \rho\left(0, y_{0}, v_{0} ; t, y, v\right) \mathrm{d} v}{\varrho\left(0, y_{0}, v_{0} ; t, y\right)}=\frac{|\Sigma|(t)}{\nu_{1}(t)}+\left(\mathbb{E}_{\left(y_{0}, v_{0}\right)}\left[\mathcal{V}_{t} / Y_{t}=y\right]\right)^{2} .
\end{aligned}
$$

Note that the conditional variance of the Langevin-Ornstein-Uhlenbeck velocity, starting from $\left(y_{0}, v_{0}\right)$ at times 0 , in other words the second moment of the turbulent velocity of this Lagrangian model, is spatially homogeneous:

$$
\operatorname{Var}_{\left(y_{0}, v_{0}\right)}\left(\mathcal{V}_{t} / Y_{t}=y\right)=\frac{|\Sigma|(t)}{\nu_{1}(t)}
$$

We consider now the case where the initial conditions $\left(Y_{0}, V_{0}\right)$ are distributed with the initial law $\mu_{0}$. The expression for $\mathbb{E}\left[\mathcal{V}_{t} / Y_{t}=y\right]$ becomes

$$
\begin{aligned}
\mathbb{E}\left[\mathcal{V}_{t} / Y_{t}=y\right] & =\frac{\int_{\mathbb{R}^{2}} \int_{\mathbb{R}} v \rho\left(0, y_{0}, v_{0} ; t, y, v\right) \mathrm{d} v \mu_{0}\left(\mathrm{~d} y_{0}, \mathrm{~d} v_{0}\right)}{\int_{\mathbb{R}^{2}} \varrho\left(0, y_{0}, v_{0} ; t, y\right) \mu_{0}\left(\mathrm{~d} y_{0}, \mathrm{~d} v_{0}\right)} \\
& =\frac{\int_{\mathbb{R}^{2}}\left(\bar{V}\left(t, v_{0}\right)+\frac{\mu(t)}{\nu_{1}(t)}\left(y-\bar{Y}\left(t, y_{0}, v_{0}\right)\right)\right) \varrho\left(0, y_{0}, v_{0} ; t, y\right) \mu_{0}\left(\mathrm{~d} y_{0}, \mathrm{~d} v_{0}\right)}{\int_{\mathbb{R}^{2}} \varrho\left(0, y_{0}, v_{0} ; t, y\right) \mu_{0}\left(\mathrm{~d} y_{0}, \mathrm{~d} v_{0}\right)} \\
& =\frac{\mathbb{E}\left[\left(\bar{V}\left(t, V_{0}\right)+\frac{\mu(t)}{\nu_{1}(t)}\left(y-\bar{Y}\left(t, Y_{0}, V_{0}\right)\right)\right) \varrho\left(0, Y_{0}, V_{0} ; t, y\right)\right]}{\varrho(t, y)} \\
& =\frac{\mu(t)}{\nu_{1}(t)} y+\frac{\left.\left.\mu(t) \bar{Y}\left(t, Y_{0}, V_{0}\right)\right) \varrho\left(0, Y_{0}, V_{0} ; t, y\right)\right]}{\nu_{1}(t)},
\end{aligned}
$$

where we have set

$$
\varrho(t, y)=\int_{\mathbb{R}^{2}} \varrho\left(0, y_{0}, v_{0} ; t, y\right) \mu_{0}\left(\mathrm{~d} y_{0}, \mathrm{~d} v_{0}\right)
$$

Similarly, for $\mathbb{E}\left[\mathcal{V}_{t}^{2} / Y_{t}=y\right]$, we have

$$
\begin{aligned}
\mathbb{E}\left[\mathcal{V}_{t}^{2} / Y_{t}=y\right] & =\frac{\int_{\mathbb{R}^{2}} \int_{\mathbb{R}^{2}} v^{2} \rho\left(0, y_{0}, v_{0} ; t, y, v\right) \mathrm{d} v \mu_{0}\left(\mathrm{~d} y_{0}, \mathrm{~d} v_{0}\right)}{\int_{\mathbb{R}^{2}} \varrho\left(0, y_{0}, v_{0} ; t, y\right) \mu_{0}\left(\mathrm{~d} y_{0}, \mathrm{~d} v_{0}\right)} \\
& =\frac{\int_{\mathbb{R}^{2}}\left[\frac{|\Sigma|(t)}{\nu_{1}(t)}+\left(\bar{V}\left(t, v_{0}\right)+\frac{\mu(t)}{\nu_{1}(t)}\left(y-\bar{Y}\left(t, y_{0}, v_{0}\right)\right)\right)^{2}\right] \varrho\left(0, y_{0}, v_{0} ; t, y\right) \mu_{0}\left(\mathrm{~d} y_{0}, \mathrm{~d} v_{0}\right)}{\int_{\mathbb{R}^{2}} \varrho\left(0, y_{0}, v_{0} ; t, y\right) \mu_{0}\left(\mathrm{~d} y_{0}, \mathrm{~d} v_{0}\right)} \\
& =\frac{|\Sigma|(t)}{\nu_{1}(t)}+\frac{\mathbb{E}\left[\left(\bar{V}\left(t, V_{0}\right)+\frac{\mu(t)}{\nu_{1}(t)}\left(y-\bar{Y}\left(t, Y_{0}, V_{0}\right)\right)\right)^{2} \varrho\left(0, Y_{0}, V_{0} ; t, y\right)\right]}{\varrho(t, y)} .
\end{aligned}
$$




\section{A.2. The case of the Gaussian initial velocity law}

We denote by $g_{\sigma}(\cdot)$ the one dimensional centred Gaussian density with given standard deviation $\sigma$.

For the purpose of Section 4, we compute

$$
\begin{aligned}
& \mathbb{E}\left(\left[\bar{V}\left(t, V_{0}\right)+\frac{\mu(t)}{\nu_{1}(t)}\left(y-\bar{Y}\left(t, Y_{0}, V_{0}\right)\right)\right] \varrho\left(0, Y_{0}, V_{0} ; t, y\right)\right) \\
\text { and } & \mathbb{E}\left(\left[\bar{V}\left(t, V_{0}\right)+\frac{\mu(t)}{\nu_{1}(t)}\left(y-\bar{Y}\left(t, Y_{0}, V_{0}\right)\right)\right]^{2} \varrho\left(0, Y_{0}, V_{0} ; t, y\right)\right),
\end{aligned}
$$

when $Y_{0}$ and $V_{0}$ are independent, and $V_{0}$ is normally distributed according to $g_{\sigma_{0}}\left(v_{0}\right) d v_{0}$.

We note that, in view of (A.4), for all $\left(y_{0}, v_{0}\right) \in \mathbb{R}^{2}$,

$\varrho\left(0, y_{0}, v_{0} ; t, y\right) g_{\sigma_{0}}\left(v_{0}\right)=g_{\sigma_{1}(t)}\left(y-y_{0}-m(t-\gamma(t))\right) g_{\sigma_{2}(t)}\left(v_{0}-\frac{\gamma(t) \sigma_{0}^{2}}{\nu_{1}(t)+\left(\gamma(t) \sigma_{0}\right)^{2}}\left(y-y_{0}-m(t-\gamma(t))\right)\right)$,

with

$$
\sigma_{1}(t):=\sqrt{\nu_{1}(t)+\left(\gamma(t) \sigma_{0}\right)^{2}} \quad \text { and } \quad \sigma_{2}(t):=\frac{\sigma_{0} \sqrt{\nu_{1}(t)}}{\sqrt{\nu_{1}(t)+\left(\gamma(t) \sigma_{0}\right)^{2}}}
$$

We then immediately obtain that

$$
\int_{\mathbb{R}} \varrho\left(0, y_{0}, v_{0} ; t, y\right) g_{\sigma_{0}}\left(v_{0}\right) \mathrm{d} v_{0}=g_{\sigma_{1}(t)}\left(y-y_{0}-m(t-\gamma(t))\right)
$$

and hence

$$
\varrho(t, y)=\mathbb{E}\left(g_{\sigma_{1}(t)}\left(y-Y_{0}-m(t-\gamma(t))\right)\right) .
$$

Moreover,

$$
\begin{aligned}
& \mathbb{E}\left[\left(\bar{V}\left(t, V_{0}\right)+\frac{\mu(t)}{\nu_{1}(t)}\left(y-\bar{Y}\left(t, Y_{0}, V_{0}\right)\right)\right) \varrho\left(0, Y_{0}, V_{0} ; t, y\right)\right] \\
& =\mathbb{E}\left[\left(V_{0} \exp (-c t)+m c \gamma(t)+\frac{\mu(t)}{\nu_{1}(t)}\left(y-Y_{0}-V_{0} \gamma(t)-m(t-\gamma(t))\right)\right) \varrho\left(0, Y_{0}, V_{0} ; t, y\right)\right] \\
& =\frac{\gamma(t) \sigma_{0}^{2} \exp (-c t)+\mu(t)}{\nu_{1}(t)+\left(\gamma(t) \sigma_{0}\right)^{2}} \mathbb{E}\left[\left(y-Y_{0}-m(t-\gamma(t))\right) g_{\sigma_{1}(t)}\left(y-Y_{0}-m(t-\gamma(t))\right)\right]+m c \gamma(t) \varrho(t, y)
\end{aligned}
$$

and

$$
\begin{aligned}
\mathbb{E} & {\left[\left(\bar{V}\left(t, V_{0}\right)+\frac{\mu(t)}{\nu_{1}(t)}\left(y-\bar{Y}\left(t, Y_{0}, V_{0}\right)\right)\right)^{2} \varrho\left(0, Y_{0}, V_{0} ; t, y\right)\right] } \\
= & \mathbb{E}\left[\left(V_{0} \exp (-c t)+m c \gamma(t)+\frac{\mu(t)}{\nu_{1}(t)}\left[y-Y_{0}-V_{0} \gamma(t)-m(t-\gamma(t))\right]\right)^{2} \varrho\left(0, Y_{0}, V_{0} ; t, y\right)\right] \\
= & \frac{\nu_{1}(t)\left(\gamma(t) \sigma_{0}\right)^{2}}{\nu_{1}(t)+\left(\gamma(t) \sigma_{0}\right)^{2}}\left(\frac{\exp (-c t)}{\gamma(t)}+\frac{\mu(t)}{\nu_{1}(t)}\right)^{2} \varrho(t, y)+\mathbb{E}\left[\left(\frac{\gamma(t) \sigma_{0}^{2} \exp (-c t)+\mu(t)}{\nu_{1}(t)+\left(\gamma(t) \sigma_{0}\right)^{2}}\left[y-Y_{0}-m(t-\gamma(t))\right]+m c \gamma(t)\right)^{2}\right. \\
& \left.\times g_{\sigma_{1}(t)}\left(y-Y_{0}-m(t-\gamma(t))\right)\right]
\end{aligned}
$$


Coming back to (A.5) and (A.6), this leads to the following semi-explicit expressions for $\mathbb{E}\left[\mathcal{V}_{t} / Y_{t}=y\right]$ and $\mathbb{E}\left[\mathcal{V}_{t}^{2} / Y_{t}=y\right]$ :

$$
\begin{aligned}
\mathbb{E}\left[\mathcal{V}_{t} / Y_{t}=y\right]= & \frac{\mu(t)}{\nu_{1}(t)} y+m c \gamma(t)-\frac{\left(\gamma(t) \sigma_{0}^{2} \exp (-c t)+\mu(t)\right)}{\nu_{1}(t)+\left(\gamma(t) \sigma_{0}\right)^{2}} m(t-\gamma(t)) \\
& +\frac{\left(\gamma(t) \sigma_{0}^{2} \exp (-c t)+\mu(t)\right)}{\nu_{1}(t)+\left(\gamma(t) \sigma_{0}\right)^{2}} \frac{\mathbb{E}\left[\left(y-Y_{0}\right) g_{\sigma_{1}(t)}\left(y-Y_{0}-m(t-\gamma(t))\right)\right]}{\varrho(t, y)}, \\
\mathbb{E}\left[\mathcal{V}_{t}^{2} / Y_{t}=y\right]= & \frac{|\Sigma|(t)}{\nu_{1}(t)}+\frac{\nu_{1}(t)\left(\gamma(t) \sigma_{0}\right)^{2}}{\nu_{1}(t)+\left(\gamma(t) \sigma_{0}\right)^{2}}\left(\frac{\exp (-c t)}{\gamma(t)}-\frac{\mu(t)}{\nu_{1}(t)}\right)^{2}+\left(\frac{\gamma(t) \sigma_{0}^{2} \exp (-c t)+\mu(t)}{\nu_{1}(t)+\left(\gamma(t) \sigma_{0}\right)^{2}}\right)^{2} \\
& \times \frac{\mathbb{E}\left[\left(y-Y_{0}-m(t-\gamma(t))+\frac{\nu_{1}(t)+\left(\gamma(t) \sigma_{0}\right)^{2}}{\gamma(t) \sigma_{0}^{2} \exp (-c t)+\mu(t)} m c \gamma(t)\right)^{2} g_{\sigma_{1}(t)}\left(y-Y_{0}-m(t-\gamma(t))\right)\right]}{\varrho(t, y)} .
\end{aligned}
$$

Remark A.1. Consider the case where the conditional position $y$ is in a compact set and $Y_{0}$ has a compact support. When $t$ tends to infinity, observe that $c \gamma(t) \simeq 1, \frac{\mu(t)}{\nu_{1}(t)} \simeq \frac{1}{2 t}$ and $\sigma_{1}(t) \simeq \frac{\sigma}{c} \sqrt{t}$. Then the Eulerian velocity of the free Langevin-Ornstein-Uhlenbeck convergences to the homogeneous value $\frac{m}{2}$, as $t \rightarrow+\infty$ :

$$
\begin{aligned}
& \mathbb{E}\left[\mathcal{V}_{t} / Y_{t}=y\right] \rightarrow \frac{m}{2} \\
& \mathbb{E}\left[\mathcal{V}_{t}^{2} / Y_{t}=y\right] \rightarrow \frac{m^{2}}{4}+\frac{\sigma^{2}}{2 c}, \text { when } t \rightarrow+\infty
\end{aligned}
$$

A.2.1. The case of zero Eulerian velocity field $(m=0)$

For the reader convenience, we give the expression (A.10) and (A.11) when $m=0$ :

$$
\begin{aligned}
\mathbb{E}\left[\mathcal{V}_{t} / Y_{t}=y\right]= & \frac{\mu(t)}{\nu_{1}(t)} y+\frac{\gamma(t) \sigma_{0}^{2} \exp (-c t)+\mu(t)}{\nu_{1}(t)+\left(\gamma(t) \sigma_{0}\right)^{2}} \frac{\mathbb{E}\left[\left(y-Y_{0}\right) g_{\sigma_{1}(t)}\left(y-Y_{0}\right)\right]}{\varrho(t, y)}, \\
\mathbb{E}\left[\mathcal{V}_{t}^{2} / Y_{t}=y\right]= & \frac{|\Sigma|(t)}{\nu_{1}(t)}+\frac{\nu_{1}(t)\left(\gamma(t) \sigma_{0}\right)^{2}}{\nu_{1}(t)+\left(\gamma(t) \sigma_{0}\right)^{2}}\left(\frac{\exp (-c t)}{\gamma(t)}-\frac{\mu(t)}{\nu_{1}(t)}\right)^{2} \\
& +\left(\frac{\gamma(t) \sigma_{0}^{2} \exp (-c t)+\mu(t)}{\nu_{1}(t)+\left(\gamma(t) \sigma_{0}\right)^{2}}\right)^{2} \frac{\mathbb{E}\left[\left(y-Y_{0}\right)^{2} g_{\sigma_{1}(t)}\left(y-Y_{0}\right)\right]}{\varrho(t, y)} .
\end{aligned}
$$

Remark A.2. Consider again the case where the conditional position $y$ is in a compact set and $Y_{0}$ has a compact support. When $t$ tends to infinity, we easily identify two constants $C_{1}^{\prime}$ and $C_{2}^{\prime}$, depending on $y$ such that

$$
\begin{aligned}
\mathbb{E}\left[\mathcal{V}_{t} / Y_{t}=y\right] & =\frac{C_{1}^{\prime}}{2 t}+o\left(\frac{1}{t}\right), \\
\mathbb{E}\left[\mathcal{V}_{t}^{2} / Y_{t}=y\right]-\frac{\sigma^{2}}{2 c} & =\frac{C_{2}^{\prime}}{t}+o\left(\frac{1}{t}\right) .
\end{aligned}
$$




\section{Appendix B. The Periodic-Langevin-Ornstein-Uhlenbeck Process}

For a fixed $L>0$, we define the torus $\mathbb{T}:=\mathbb{R} / L \mathbb{Z}$. Hereafter, we consider the periodic Langevin-OrnsteinUhlenbeck process $\left(Y^{\mathbb{T}}, \mathcal{V}\right)$ on $\mathbb{T} \times \mathbb{R}$ which is the solution to the following SDEs:

$$
\left\{\begin{array}{l}
Y_{t}^{\mathbb{T}}=\left[Y_{0}^{\mathbb{T}}+\int_{0}^{t} \mathcal{V}_{s} \mathrm{~d} s\right] \bmod L \\
\mathcal{V}_{t}=V_{0}-c \int_{0}^{t}\left(\mathcal{V}_{s}-m\right) \mathrm{d} s+\sigma W_{t}
\end{array}\right.
$$

for $[x] \bmod L:=x-L\lfloor x / L\rfloor$ where $\lfloor x\rfloor$ is the integer part of $x \in \mathbb{R}$. Equation (B.14) is considered on a probability space $(\Omega, \mathcal{F}, \mathbb{P})$, equipped with a one dimensional Brownian motion $W$ and initial position and velocity condition $\left(Y_{0}^{\mathbb{T}}, V_{0}\right) \mu_{0}$-distributed in $[0, L] \times \mathbb{R}$. We now compute the explicit expressions for the space marginal law of $\left(Y_{t}^{\mathbb{T}}, \mathcal{V}_{t}\right)$, and for the conditional moments $\mathbb{E}\left[\mathcal{V}_{t} / Y_{t}^{\mathbb{T}}=y\right]$ and $\mathbb{E}\left[\mathcal{V}_{t}^{2} / Y_{t}^{\mathbb{T}}=y\right]$. First, let us notice that the process $\left(Y_{t}^{\mathbb{T}}, \mathcal{V}_{t}\right)$ writes as

$$
\left(Y_{t}^{\mathbb{T}}, \mathcal{V}_{t}\right)=\left(\left[Y_{t}\right] \bmod L, \mathcal{V}_{t}\right)
$$

for $\left(Y_{t}, \mathcal{V}_{t}\right)$ solution to $(\mathrm{A} .1)$. Hence, for all $f \in \mathcal{C}_{b}([0, L] \times \mathbb{R})$,

$$
\mathbb{E}\left[f\left(Y_{t}^{\mathbb{T}}, \mathcal{U}_{t}\right)\right]=\mathbb{E}\left[f\left(\left[Y_{t}\right] \bmod L, \mathcal{U}_{t}\right)\right]=\mathbb{E}\left[f\left(Y_{t}-L\left\lfloor Y_{t} / L\right\rfloor, \mathcal{U}_{t}\right)\right]=\sum_{k \in \mathbb{Z}} \mathbb{E}_{\mathbb{P}}\left[f\left(Y_{t}-L k, \mathcal{U}_{t}\right) \mathbb{1}_{\left\{Y_{t} \in[L k, L(k+1))\right\}}\right]
$$

Therefore, the density $\rho^{\mathbb{T}}\left(0, y_{0}, v_{0} ; t, y, v\right)$, of the law of $\left(Y_{t}^{\mathbb{T}}, \mathcal{V}_{t}\right)$ starting from $\left(y_{0}, v_{0}\right)$ is given by

$$
\rho^{\mathbb{T}}\left(0, y_{0}, v_{0} ; t, y, v\right):=\sum_{k \in \mathbb{Z}} \rho\left(0, y_{0}, v_{0} ; t, y+k L, v\right) \text { for a.e. }(y, v) \in[0, L] \times \mathbb{R}
$$

where the density $\rho\left(0, y_{0}, v_{0} ; t, y+k L, v\right)$ of the law of $\left(Y_{t}, \mathcal{V}_{t}\right)$ starting from $\left(y_{0}, v_{0}\right)$ is explicitly written in (A.3). Now we compute the conditional moments, for all $(t, y) \in(0,+\infty) \times[0, L]$,

$$
\begin{aligned}
\int_{[0, L] \times \mathbb{R}} \int_{\mathbb{R}} v^{\alpha} \rho^{\mathbb{T}}\left(0, y_{0}, v_{0} ; t, y, v\right) \mathrm{d} v \mu_{0}\left(\mathrm{~d} y_{0}, \mathrm{~d} v_{0}\right)= & \int_{[0, L] \times \mathbb{R}} \sum_{k \in \mathbb{Z}}\left(\int_{\mathbb{R}} v^{\alpha} \rho\left(0, y_{0}, v_{0} ; t, y+k L, v\right) \mathrm{d} v\right) \\
& \times \mu_{0}\left(\mathrm{~d} y_{0}, \mathrm{~d} v_{0}\right), \forall \alpha>0 .
\end{aligned}
$$

Again, we assume that $Y_{0}$ and $V_{0}$ are independent, $V_{0}$ is normally distributed according to $g_{\sigma_{0}}\left(v_{0}\right) \mathrm{d} v_{0}$, and we denote by $\zeta_{0}\left(\mathrm{~d} y_{0}\right)$ the law of $Y_{0}$.

Combining (B.15), (B.16) with the identities (A.5), (A.6), and (A.8), (A.9), we get

$$
\begin{aligned}
& \varrho^{\mathbb{T}}(t, y)=\int_{\mathbb{R}} \rho^{\mathbb{T}}\left(0, y_{0}, v_{0} ; t, y, v\right) \mu_{0}\left(\mathrm{~d} y_{0}, \mathrm{~d} v_{0}\right)=\sum_{k \in \mathbb{Z}} \int_{0}^{L} g_{\sigma_{1}(t)}\left(y+k L-y_{0}-m(t-\gamma(t))\right) \zeta_{0}\left(\mathrm{~d} y_{0}\right) \\
& \int_{[0, L] \times \mathbb{R}} \int_{\mathbb{R}} v \rho^{\mathbb{T}}\left(0, y_{0}, v_{0} ; t, y, v\right) \mathrm{d} v \mu_{0}\left(\mathrm{~d} y_{0}, \mathrm{~d} v_{0}\right)=m c \gamma(t) \varrho^{\mathbb{T}}(t, y)+\frac{\left.\gamma(t) \sigma_{0}^{2} \exp (-c t)+\mu(t)\right)}{\nu_{1}(t)+\left(\gamma(t) \sigma_{0}\right)^{2}} \\
& \quad \times \sum_{k \in \mathbb{Z}} \int_{0}^{L}\left(y+k L-y_{0}-m(t-\gamma(t))\right) g_{\sigma_{1}(t)}\left(y+k L-y_{0}-m(t-\gamma(t))\right) \zeta_{0}\left(\mathrm{~d} y_{0}\right)
\end{aligned}
$$


and

$$
\begin{aligned}
& \int_{[0, L] \times \mathbb{R}} \int_{\mathbb{R}} v^{2} \rho^{\mathbb{T}}\left(0, y_{0}, v_{0} ; t, y, v\right) \mathrm{d} v \mu_{0}\left(\mathrm{~d} y_{0}, \mathrm{~d} v_{0}\right)=\frac{|\Sigma|(t)}{\nu_{1}(t)} \varrho^{\mathbb{T}}(t, y)+\frac{\nu_{1}(t)\left(\gamma(t) \sigma_{0}\right)^{2}}{\nu_{1}(t)+\left(\gamma(t) \sigma_{0}\right)^{2}}\left(\frac{\exp (-c t)}{\gamma(t)}-\frac{\mu(t)}{\nu_{1}(t)}\right)^{2} \varrho^{\mathbb{T}}(t, y) \\
& +\sum_{k \in \mathbb{Z}} \int_{0}^{L}\left(\frac{\gamma(t) \sigma_{0}^{2} \exp (-c t)+\mu(t)}{\nu_{1}(t)+\left(\gamma(t) \sigma_{0}\right)^{2}}\left[y+k L-y_{0}-m(t-\gamma(t))\right]+m c \gamma(t)\right)^{2} g_{\sigma_{1}(t)}\left(y+k L-y_{0}-m(t-\gamma(t))\right) \zeta_{0}\left(\mathrm{~d} y_{0}\right) .
\end{aligned}
$$

In the particular case where $Y_{0}^{\mathbb{T}}$ is uniformly distributed in $[0, L]\left(i . e . \zeta_{0}\left(\mathrm{~d} y_{0}\right)=\frac{1}{L} \mathrm{~d} y_{0}\right)$, one has

$$
\sum_{k \in \mathbb{Z}} \int_{0}^{L} g_{\sigma_{1}(t)}\left(y+k L-y_{0}-m(t-\gamma(t))\right) \zeta_{0}\left(\mathrm{~d} y_{0}\right)=\frac{1}{L} \sum_{k \in \mathbb{Z}} \int_{0}^{L} g_{\sigma_{1}(t)}\left(y+k L-y_{0}-m(t-\gamma(t))\right) \mathrm{d} y_{0}=\frac{1}{L}
$$

from which one deduces that for all $t \in(0,+\infty)$

$$
\begin{aligned}
& \varrho^{\mathbb{T}}(t, y)=\frac{1}{L}, \\
& \int_{[0, L] \times \mathbb{R}} \int_{\mathbb{R}} v \rho^{\mathbb{T}}\left(0, y_{0}, v_{0} ; t, y, v\right) \mathrm{d} v \mu_{0}\left(\mathrm{~d} y_{0}, \mathrm{~d} v_{0}\right)=\frac{1}{L} m c \gamma(t),
\end{aligned}
$$

and hence

$$
\mathbb{E}\left[\mathcal{V}_{t} / Y_{t}^{\mathbb{T}}=y\right]=m(1-\exp (-c t))
$$

Similarly

$$
\begin{gathered}
\int_{[0, L] \times \mathbb{R}} \int_{\mathbb{R}} v^{2} \rho^{\mathbb{T}}\left(0, y_{0}, v_{0} ; t, y, v\right) \mathrm{d} v \mu_{0}\left(\mathrm{~d} y_{0}, \mathrm{~d} v_{0}\right)=\frac{1}{L}\left(\frac{|\Sigma|(t)}{\nu_{1}(t)}+\frac{\nu_{1}(t)\left(\gamma(t) \sigma_{0}\right)^{2}}{\nu_{1}(t)+\left(\gamma(t) \sigma_{0}\right)^{2}}\left(\frac{\exp (-c t)}{\gamma(t)}-\frac{\mu(t)}{\nu_{1}(t)}\right)^{2}\right) \\
+\sum_{k \in \mathbb{Z}} \frac{1}{L} \int_{0}^{L}\left\{\frac{\gamma(t) \sigma_{0}^{2} \exp (-c t)+\mu(t)}{\nu_{1}(t)+\left(\gamma(t) \sigma_{0}\right)^{2}}\left[y+k L-y_{0}-m(t-\gamma(t))\right]-m c \gamma(t)\right\}^{2} \\
\times g_{\sigma_{1}(t)}\left(y+k L-y_{0}-m(t-\gamma(t))\right) \mathrm{d} y_{0}
\end{gathered}
$$

and then

$$
\mathbb{E}\left[\mathcal{V}_{t}^{2} / Y_{t}^{\mathbb{T}}=y\right]=\frac{|\Sigma|(t)}{\nu_{1}(t)}+\frac{\nu_{1}(t)\left(\gamma(t) \sigma_{0}\right)^{2}}{\nu_{1}(t)+\left(\gamma(t) \sigma_{0}\right)^{2}}\left(\frac{\exp (-c t)}{\gamma(t)}-\frac{\mu(t)}{\nu_{1}(t)}\right)^{2}+(m c \gamma(t))^{2}+\frac{\left(\gamma(t) \sigma_{0}^{2} \exp (-c t)+\mu(t)\right)^{2}}{\nu_{1}(t)+\left(\gamma(t) \sigma_{0}\right)^{2}}
$$

The asymptotic behaviour of the first and second conditional moments of velocity is then obvious: $\mathbb{E}\left[\mathcal{V}_{t} / Y_{t}^{\mathbb{T}}=y\right]$ tends to $m$ exponentially fast, and

$$
\mathbb{E}\left[\mathcal{V}_{t}^{2} / Y_{t}^{\mathbb{T}}=y\right] \rightarrow m^{2}+\frac{\sigma^{2}}{2 c} \text { when } t \rightarrow+\infty
$$

We end this subsection with some remarks on the asymptotic behaviour of the solution to (B.14). If it exists, the invariant measure associated to this process is a solution to the stationary PDE

$$
v \partial_{y} \rho_{\infty}(y, v)-c \partial_{v}\left((v-m) \rho_{\infty}(y, v)\right)-\frac{\sigma^{2}}{2} \partial_{v}^{2} \rho_{\infty}(y, v)=0, \text { for }(y, v) \in[0, L] \times \mathbb{R}
$$


with periodic boundary condition, $\rho_{\infty}(0, v)=\rho_{\infty}(L, v)$ in its position variable, and such that $\left(1+|v|^{2}\right) \rho_{\infty}(y, v)+$ $(1+|v|)\left|\partial_{v} \rho_{\infty}(y, v)\right|$ decays to zero when $v$ tends to infinity, uniformly in $y \in[0, L]$. This limit equation admits smooth solutions, and it is easy to check that any solution is such that

$$
\int_{\mathbb{R}} v \rho_{\infty}(y, v) \mathrm{d} v=\frac{m}{L} .
$$

Indeed, integrating (B.17) over the velocity space, one can check that $y \mapsto \int_{\mathbb{R}} v \rho_{\infty}(y, v) \mathrm{d} v$ is equal to some constant $C$. Multiplying (B.17) by $v$ and integrating the resulting equation over position and velocity spaces, one gets

$$
0=\left(\int_{\mathbb{R}}|v|^{2} \rho_{\infty}(L, v) \mathrm{d} v-\int_{\mathbb{R}}|v|^{2} \rho_{\infty}(0, v) \mathrm{d} v\right)+c\left(C L-m \int_{[0, L] \times \mathbb{R}} \rho_{\infty}(y, v) \mathrm{d} y \mathrm{~d} v\right)
$$

Using the periodic condition at the boundary, we identify $C=m / L$. We also note that an explicit solution to equation (B.17) is

$$
\rho_{\infty}(y, v)=\frac{\sqrt{c}}{L \sqrt{\pi \sigma^{2}}} \exp \left(-\frac{c|v-m|^{2}}{\sigma^{2}}\right) .
$$

Acknowledgements. This work was partially supported by the French Agence de l'Environnement et de la Maîtrise de l'Énergie and Agence Nationale de la Recherche under Grant No. ANR-08-SYSCOMM-014. The fourth author was supported by the FONDAP-CMM program in Applied Mathematics and by Fondecyt Postdoctoral Project No. 3100132.

\section{REFERENCES}

[1] F. Bernardin, M. Bossy, C. Chauvin, P. Drobinski, A. Rousseau and T. Salameh, Stochastic downscaling methods: application to wind refinement. Stoch. Environ. Res. Risk. Assess. 23 (2009) 851-859.

[2] M. Bossy, J.-F. Jabir and D. Talay, On conditional McKean Lagrangian stochastic models. Research report RR-6761, INRIA, France (2008) http://hal.inria.fr/inria-00345524/en/.

[3] M. Bossy, J. Fontbona and J.-F. Jabir, Incompressible Lagrangian stochastic model in the torus. In preparation.

[4] J.A. Carrillo, Global weak solutions for the initial-boundary-value problems to the Vlasov-Poisson-Fokker-Planck system. Math. Meth. Appl. Sci. 21 (1998) 907-938.

[5] C. Cercignani, The Boltzmann equation and its applications, Applied Mathematical Sciences 67. Springer-Verlag, New York (1988).

[6] C. Chauvin, S. Hirstoaga, P. Kabelikova, F. Bernardin and A. Rousseau, Solving the uniform density constraint in a downscaling stochastic model. ESAIM: Proc. 24 (2008) 97-110.

[7] C. Chauvin, F. Bernardin, M. Bossy and A. Rousseau, Wind simulation refinement: some new challenges for particle methods, in Springer Mathematics in Industry series, ECMI (to appear).

[8] P. Degond, Global existence of smooth solutions for the Vlasov-Fokker-Planck equation in 1 and 2 space dimensions. Ann. Sci. École Norm. Sup. 19 (1986) 519-542.

[9] P. Degond and S. Mas-Gallic, Existence of solutions and diffusion approximation for a model Fokker-Planck equation. Internal report, École Polytechnique, Palaiseau, France (1985).

[10] M. Di Francesco and A. Pascucci, On a class of degenerate parabolic equations of Kolmogorov type. AMRX Appl. Math. Res. Express 3 (2005) 77-116.

[11] M. Di Francesco and S. Polidoro, Schauder estimates, Harnack inequality and Gaussian lower bound for Kolmogorov-type operators in non-divergence form. Adv. Diff. Equ. 11 (2006) 1261-1320.

[12] P. Drobinski, J.L. Redelsperger and C. Pietras, Evaluation of a planetary boundary layer subgrid-scale model that accounts for near-surface turbulence anisotropy. Geophys. Res. Lett. 33 (2006) L23806.

[13] C.W. Gardiner, Handbook of stochastic methods, Springer Series in Synergetics 13. Second edition, Springer-Verlag (1985).

[14] J.-L. Guermond and L. Quartapelle, Calculation of incompressible viscous flows by an unconditionally stable projection FEM. J. Comput. Phys. 132 (1997) 12-33.

[15] F.H. Harlow and P.I. Nakayama, Transport of turbulence energy decay rate. Technical report (1968) 451.

[16] J.-F. Jabir, Lagrangian Stochastic Models of conditional McKean-Vlasov type and their confinements. Ph.D. Thesis, University of Nice-Sophia-Antipolis, France (2008).

[17] I. Karatzas and S.E. Shreve, Brownian Motion and Stochastic Calculus. Springer-Verlag, New York (1988). 
[18] A. Lachal, Les temps de passage successifs de l'intégrale du mouvement brownien. Ann. I.H.P. Probab. Stat. 33 (1997) 1-36.

[19] E. Lanconelli, A. Pascucci and S. Polidoro, Linear and nonlinear ultraparabolic equations of Kolmogorov type arising in diffusion theory and in finance, in Nonlinear problems in mathematical physics and related topics, Int. Math. Ser., Kluwer/Plenum, New York (2002) 243-265.

[20] H.P. McKean, Jr, A winding problem for a resonator driven by a white noise. J. Math. Kyoto Univ. 2 (1963) $227-235$.

[21] J.-P. Minier and E. Peirano, The pdf approach to turbulent polydispersed two-phase flows. Phys. Rep. 352 (2001) 1-214.

[22] B. Mohammadi and O. Pironneau, Analysis of the $k$-epsilon turbulence model. Masson, Paris (1994).

[23] C.M. Mora, Weak exponential schemes for stochastic differential equations with additive noise. IMA J. Numer. Anal. 25 (2005) 486-506.

[24] T. Plewa, T. Linde and V.G. Weirs Eds., Adaptive Mesh Refinement - Theory and Applications, Lecture Notes in Computational Science and Engineering 41. Springer, Chicago (2003).

[25] S.B. Pope, P.D.F. methods for turbulent reactive flows. Prog. Energy Comb. Sci. 11 (1985) 119-192.

[26] S.B. Pope, On the relationship between stochastic Lagrangian models of turbulence and second-moment closures. Phys. Fluids 6 (1993) 973-985.

[27] S.B. Pope, Lagrangian pdf methods for turbulent flows. Annu. Rev. Fluid Mech. 26 (1994) 23-63.

[28] S.B. Pope, Turbulent flows. Cambridge Univ. Press, Cambridge (2003).

[29] P.-A. Raviart, An analysis of particle methods, in Numerical methods in fluid dynamics, Lecture Notes in Mathematics 1127, Springer, Berlin (1985) 243-324.

[30] J.L. Redelsperger, F. Mahé and P. Carlotti, A simple and general subgrid model suitable both for surface layer and free-stream turbulence. Bound. Layer Meteor. 101 (2001) 375-408.

[31] A. Rousseau, F. Bernardin, M. Bossy, P. Drobinski and T. Salameh, Stochastic particle method applied to local wind simulation, in Proc. IEEE International Conference on Clean Electrical Power (2007) 526-528.

[32] P. Sagaut, Large eddy simulation for incompressible flows - An introduction. Scientific Computation, Springer-Verlag, Berlin (2001).

[33] D.W. Stroock and S.R. Varadhan, Multidimensional diffusion processes. Springer-Verlag, Berlin (1979).

[34] R.B. Stull, An Introduction to Boundary Layer Meteorology. Atmospheric and Oceanographic Sciences Library, Kluwer Academic Publishers (1988).

[35] A.-S. Sznitman, Topics in propagation of chaos, in École d'Été de Probabilités de Saint-Flour XIX - 1989, Lecture Notes in Mathematics 1464, Springer, Berlin (1991) 165-251. 